\title{
Analyzing and Securing SOME/IP Automotive Services with Formal and Practical Methods
}

\author{
Daniel Zelle \\ daniel.zelle@sit.fraunhofer.de \\ Fraunhofer Institute for Secure Information Technology \\ Darmstadt, Germany \\ Dustin Kern \\ dustin.kern@h-da.de \\ Darmstadt University of Applied Sciences \\ Darmstadt, Germany
}

\author{
Timm Lauser \\ timm.lauser@h-da.de \\ Darmstadt University of Applied Sciences \\ Darmstadt, Germany \\ Christoph Krauß ${ }^{*}$ \\ christoph.krauss@h-da.de \\ Darmstadt University of Applied Sciences \\ Darmstadt, Germany
}

\begin{abstract}
Automotive Ethernet is increasingly used in modern vehicles and complements or replaces legacy bus systems such as CAN. Ethernet also enables service-oriented communication with the Scalable service-Oriented MiddlewarE over IP (SOME/IP) middleware. In this paper, we present a formal and practical security analysis of SOME/IP, the identified Man-in-the-Middle (MITM) attacks, and propose two security extensions. The attacks are possible even if SOME/IP is used in combination with link layer security mechanisms. The attacker can impersonate a service offering server and a service consuming client. The two most common communication methods, request/response and publish/subscribe, are both vulnerable. In most communication scenarios, we are able to route all messages over the attacker. Our security extensions for authentication and authorization of service provisioning and usage protect against these attacks. We formally analyze the security and evaluate the overhead with practical implementations.
\end{abstract}

\section{CCS CONCEPTS}

-Security and privacy $\rightarrow$ Security protocols; Embedded systems security; • Applied computing $\rightarrow$ Transportation.

\section{KEYWORDS}

Automotive Security, SOME/IP, Man-in-the-Middle Attack

\section{ACM Reference Format:}

Daniel Zelle, Timm Lauser, Dustin Kern, and Christoph Krauß. 2021. Analyzing and Securing SOME/IP Automotive Services with Formal and Practical Methods. In The 16th International Conference on Availability, Reliability and Security (ARES 2021), August 17-20, 2021, Vienna, Austria. ACM, New York, NY, USA, 20 pages. https://doi.org/10.1145/3465481.3465748

*Also with Fraunhofer Institute for Secure Information Technology.

Permission to make digital or hard copies of part or all of this work for personal or classroom use is granted without fee provided that copies are not made or distributed for profit or commercial advantage and that copies bear this notice and the full citation on the first page. Copyrights for third-party components of this work must be honored

For all other uses, contact the owner/author(s).

ARES 2021, August 17-20, 2021, Vienna, Austria

(C) 2021 Copyright held by the owner/author(s).

ACM ISBN 978-1-4503-9051-4/21/08.

https://doi.org/10.1145/3465481.3465748

\section{INTRODUCTION}

A major technology shift is currently taking place in vehicles, e.g., to implement new infotainment functionalities or advanced driver assistance systems, which are also required for autonomous driving. These functionalities require high and flexible communication bandwidths and are more and more realized using Automotive Ethernet (e.g., BroadR-Reach) and IP-based communication. In contrast to the Controller Area Network (CAN) bus, which provides only static communication, the use of Ethernet and IP enables dynamic and service-oriented communication with efficient bandwidth use. The Scalable service-Oriented MiddlewarE over IP (SOME/IP) can be used as an automotive middleware solution supporting remote procedure calls, event notifications, and the underlying serialization/wire format. SOME/IP has been developed to address automotive requirements (e.g., integration in AUTomotive Open System ARchitecture (AUTOSAR) or fast response times) and supports devices of different sizes and operating systems. Electronic Control Units (ECUs) use SOME/IP to announce offered services (as a server) or make use of a service (as a client). Since the SOME/IP specification does not consider any security mechanisms, it is often used in combination with link layer security mechanisms such as MACsec or AUTOSAR Secure Onboard Communication (SecOC).

This paper has two main contributions. First, we introduce MITM attacks on SOME/IP which are even possible when link layer security mechanisms are deployed. If an attacker has compromised a single ECU of the Electrical / Electronic (E/E) system of the vehicle (either by physical access or remotely like in the Jeep hack [23]), the attacker can impersonate both, a SOME/IP server and a client to direct the communication through the attacker. For this, we present a formal analysis with the Tamarin prover $^{1}$, which we used to identify the MITM attacks, and a practical evaluation with the open-source reference SOME/IP implementation vsomeip [7] and the automotive development and testing tool CANoe [11]. Second, we propose two SOME/IP protocol extensions for securing SOME/IP service discovery and all subsequent SOME/IP communication. Our solutions enable authentication and authorization of the provision and use of SOME/IP services. Our first approach initially uses certificates and digital signatures for establishing symmetric keys used to secure the subsequent SOME/IP communication. The second approach uses only efficient symmetric cryptography but

\footnotetext{
${ }^{1}$ https://tamarin-prover.github.io/
} 
requires an additional authorization server (AS) in the E/E architecture. Both approaches restrict service provisioning and usage, thus, limiting the damage an attacker can cause by compromising an ECU. We formally analyze the security of both approaches with the Tamarin prover and evaluate the introduced additional overhead with our practical implementation.

The paper is structured as follows: In Section 2, we discuss related work. An overview of SOME/IP is given in Section 3. Section 4 introduces our formal security analysis approach which we used to identify the MITM attacks described in Section 5. Implementation and evaluation of the attacks are described in Section 6. In Section 7, we describe our two security extensions for SOME/IP, their formal analysis, and the practical evaluation. Finally, we conclude the paper in Section 8.

\section{RELATED WORK}

Complex MITM attacks were not relevant for in-vehicle networks using classical automotive bus systems such as the CAN since routing is necessary in a bus system. Thus, previous work on in-vehicle attacks focused on replay or injection attacks on CAN and other bus systems. Koscher et al. presented attacks enabling brake and acceleration control via CAN in [17]. How to send messages directly or indirectly to in-vehicle networks via OBD-II, CD, WiFi, Bluetooth, or cellular has been investigated in [9]. Miller and Valasek showed in [22] how to control the steering, braking, acceleration, and the display of a car via the CAN network. They also presented an attack to remotely control a car in [23].

To secure the communication in automotive E/E architectures, several approaches have been proposed. SecOC [1] is standardized by AUTOSAR for securing CAN communication with symmetric cryptography. The security of SecOC has been formally analyzed in [19]. With increasing computing power and higher bandwidth also asymmetric cryptography can be used. For example, TLS is part of AUTOSAR (both classic and adaptive platform) and has been discussed in the literature, e.g., in [31]. IPsec is also supported by the adaptive platform. TLS 1.3 has also been formally analyzed using Tamarin [10], as well as ProVerif [5]. Tamarin has been also used to analyze V2X revocation protocols [29] and an electric vehicle charging protocol [20].

Securing SOME/IP was discussed in [18]. They propose a central entity for the key material distribution using (D)TLS during the event group subscription. The service offer and find offer are not protected. SOME/IP itself is extended with a TESLA protocol allowing broadcast communication. Using TESLA with a delayed authentication (cf. [25]) for broadcast messages has the drawback that the SOME/IP process flow needs to be changed (for caching messages until the verification messages arrive) and that the introduced latency is not suitable for time-critical data.

To detect attacks on SOME/IP, Herold et al. present an Intrusion Detection System (IDS) for SOME/IP based on Complex Event Processing (CEP) in [12]. The IDS can detect attacks from the following groups: malformed packages, protocol violations, and timing problems. This approach has the same problems as other IDS approaches, namely false-positive and false-negative results.

Iorio et al. propose a security framework to protect SOME/IP communications [15]. Initially, individual handshakes between each client requesting a service and the service offering server using certificates and asymmetric cryptography are executed for establishing a shared symmetric key. This key is used for securing subsequent messages. The main drawbacks are similar to TLS, i.e., the introduced overhead for asymmetric cryptography and that broadcast messages, for service offer messages, are not possible anymore. Instead, "find service" messages are sent to perform an individual key exchange.

Securing service-oriented communication is also discussed in other areas. For example, to secure the Robot Operating System (ROS) the fork Secure ROS [27] enables secure communication among ROS nodes by setting up an IPsec connection and restricting service providers and requesters by predefining allowed IP addresses. However, this approach contradicts the idea of a dynamic network and service discovery.

\section{SOME/IP}

The Scalable service-Oriented MiddlewarE over IP (SOME/IP) [3] is a middleware specification designed for transmission and serialization of control signals, especially in automotive use cases. SOME/IP resides on top of an (automotive) TCP/IP or UDP/IP stack and provides an abstract service-oriented interface for applications (cf. Figure 1). Thus, an application does not need to handle IP addresses and ports but services instead. The main goal of SOME/IP is the realization of flexible and bandwidth-efficient communication.

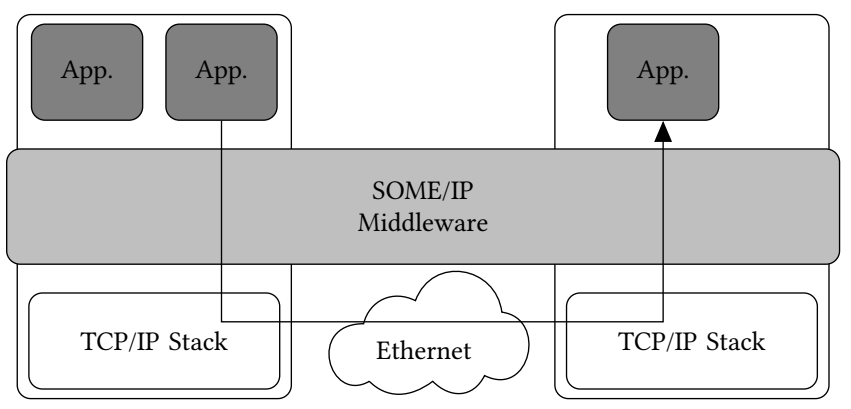

Figure 1: SOME/IP Architecture

SOME/IP can be roughly divided into three parts: Service Discovery (SD), Remote Procedure Call (RPC), and access to process data. SD enables server ECUs to publish services and client ECUs to subscribe to services in the vehicle network which are identified by a Service $I D$ and, if required, additional options such as the endpoint option (i.e., IP-address, transport protocol (UDP/TCP), and port number). Multiple instances of a service (with the same Service ID) are possible in a network and are uniquely identified by different Instance IDs. The offered services can be accessed using RPC and it is possible to get notifications about events. Process data can be accessed by applications using set and get.

SOME/IP-SD is used to locate service instances, detect if service instances are running, and implements a publish/subscribe handling [2]. For offering a service instance, a server sends a multicast service offer message OfferService(Service ID, Instance ID) with optional options such as the above-mentioned endpoint options. The server can stop offering a service instance by sending a Stop0ffer(Service ID, Instance ID) message. If clients 
do not receive suitable service offer messages with the required Service ID, they can actively send a FindService(Service ID, Instance ID) message which can be answered by servers offering the requested service. The Instance ID can be either set to a specific value or $0 x F F F F$, if all service instances shall be found. The publish/subscribe mechanism can be used in cases in which a client requires a set of signals from a server but does not want to request that information manually each time it is required. A client subscribes to an event group with a SubscribeEventgroup(Service ID, Instance ID) confirmed with SubscribeEventgroupACK ( Service ID, Instance ID) by the server. A client unsubscribes with a StopSubscribeEventgroup(Service ID, Instance ID) message. While a subscription is active, the server regularly sends event messages to the client. Additionally, SOME/IP provides a load balancing option for service instances. A server can set a priority and a weight in its service offer. A client shall select the service with the highest priority. In case there are multiple service instances with the same priority, the service instance shall be chosen randomly based on the weights of the service instances.

For RPC, SOME/IP provides several communication patterns. The most common pattern is the request/response pattern, where the client sends a request message, which is answered by the server. Requests without response are called fire\&forget. Notification events are sent regularly from a server to a client if the client has subscribed to a service using SOME/IP-SD. In certain cases, the server sends the client a notification event, e.g., an updated value or an event that occurred. SOME/IP is used for transporting the event data and SOME/IP-SD is used for publishing and subscribing.

Both, the SOME/IP Protocol Specification [3] and the SOME/IPSD Protocol Specification [2] do not really consider security aspects (cf. Section 2). Since SOME/IP does not provide any security mechanisms, attacks are obviously possible. For this reason, vehicles lately introduce network security protocols like Media Access Control Security (MACsec) [13, 14] or AUTOSAR's SecOC [1]. Thus, ECUs accept only authentic messages from other ECUs, e.g., attackers with physical access cannot inject messages to the network.

SOME/IP is typically used in Automotive Ethernet networks which replace parts of classical vehicle network technology for example CAN bus, MOST or FlexRay. Using Ethernet, the network topology also changes from a bus to a star system with several switches. According to [26], the first generation of Automotive Ethernet networks using SOME/IP consist of few switches and a low two-digit number of Ethernet-ECUs where each ECU offers a few tens of services.

\section{FORMAL SECURITY ANALYSIS APPROACH}

In this section, we outline our formal security analysis approach used to evaluate SOME/IP and our proposed security extensions.

\subsection{Symbolic Model}

We analyze the security of SOME/IP and our proposed extensions in the symbolic model, also called Dolev-Yao model. In this model, cryptographic primitives are assumed to be perfectly secure, i.e., the attacker cannot break their security properties. For example, encrypted messages can only be decrypted when in possession of the corresponding decryption key. Thus, security analysis in this model focuses on the composition of these primitives. This simplification makes the model more suitable for automation and there are powerful tools available supporting the verification of security properties within this model. Despite this simplification, analyses in the symbolic model are very powerful and enable the identification of most practical attacks on protocols (cf. Section 2).

Since SOME/IP does not provide any security mechanisms, we restrict the capabilities of the attacker in the analysis of SOME/IP by assuming the use of an underlying authentication protocol, such as $\mathrm{SecOC}$ or MACsec. However, we allow the attacker to compromise ECUs. As the multitude of attacks such as the Jeep hack [23], the Mercedes-Benz hack [30], attacks on BMW [8], the hack of Volkswagen infotainment systems [16] and the attacks on Tesla Model $\mathrm{S}$ [24] show, compromised ECUs are a real and serious threat. For the analysis of our proposed security extensions, we assume the powerful Dolev-Yao attacker with full control over the network, which can also compromise ECUs.

\subsection{Security Properties in the Symbolic Model}

The focus of our analysis is authentication, which is required to prevent unauthorized manipulation of the SOME/IP communication, especially MITM attacks. We use the authentication properties defined by Lowe [21]. They form a hierarchy, starting with the relatively weak aliveness property and going up to the much stronger notion of injective agreement.

Applied to a message authentication protocol, aliveness requires that whenever a message is received and accepted by a protocol entity, the entity it assumes to be the sender has previously sent a message. However, that message is not required to be identical to the received message. As the name implies, this property only verifies that the supposed sender was alive, that it was previously running the same protocol with the corresponding role.

We use the injective agreement property in the verification of our proposed security extensions (cf. Section 7.3). In essence, the property requires that whenever a message is received and accepted, exactly the same message has been previously sent to the recipient by the claimed sender. Moreover, the message can only be accepted once by the recipient, therefore, message replay by the attacker is not possible. Thus, providing injective agreement is an effective countermeasure against MITM attacks. While injective agreement is a very strong authentication property, it does not consider broadcast communication patterns. Lauser et al. [19] proposed One-time group agreement as an adaptation of this property to analyze protocols that allow broadcast communication in a shared-key setting, such as SecOC which they analyze in their paper. The property requires that whenever a message is received and accepted, it has been previously sent by a legitimate sender and is only accepted once by each recipient. Therefore, the property is slightly weaker than injective agreement, as it does not verify that the message was intended for the recipient. Moreover, it does not distinguish between individual senders, and thus, does not provide suitable guarantees if the attacker model considers compromised protocol entities.

In addition, we require syntactic secrecy for the secrets used within our proposed extensions. It requires that there exists no valid trace of the model, in which the secret is learned by the attacker. Forward secrecy additionally requires that the secret remains 
unknown to the attacker, even if the long-term credentials of the involved parties are leaked after the protocol run.

\subsection{Tamarin Prover}

The Tamarin prover $[4,28]$ is a well-established tool for formal analysis in the symbolic model. It can automatically generate proofs or counterexamples for security properties based on a model of the protocol, considering an unbounded number of protocol entities and sessions. Protocol models are specified as rules for a multiset rewriting system. The state of the system is represented by facts that are manipulated through these rules. Hereby, facts in the premise of a rule are consumed and replaced by the facts in the conclusion. Moreover, rules can be labeled with action facts for referencing in security lemmas and their applicability can require input verification by adding restrictions. Security properties are specified using a subset of first-order logic over action facts and time points. However, due to the undecidability of the underlying problem, Tamarin may not terminate and manual intervention or optimization, such as intermediate security lemmas, may be required.

\section{SOME/IP ANALYSIS AND ATTACKS}

We use Tamarin to formally analyze the security of SOME/IP which is used with some link layer security mechanism. The complete Tamarin model is given in Appendix A.1.

We assume that ECUs communicate via group authenticated channels with replay protection and focus on the authentication of service discovery messages. As expected, we can prove strong authentication properties given that no legitimate ECU has been compromised, especially One-time group agreement. In this case, the attacker cannot inject valid offerService messages. However, in the presence of a stronger attacker that may compromise an ECU, authentication of messages is no longer ensured, even considering the relatively weak aliveness property, which would be implied by all other authentication properties we consider (cf. Section 4.2):

Definition 5.1 (Aliveness w.r.t. OfferService). For an honest server $S$ and an honest client $C$, we require that whenever $C$ accepts an offerService message $m$ as coming from $S, S$ has previously been active.

In Tamarin's syntax, the lemma looks as follows, where E_C_ ReceiveofferService is the event that client received an Offer Service message, supposedly from server.E_S_Created describes the event that server has been created by a rule and, thus, has been active. The nonce parameter is used by other lemmas to distinguish between individual offerService messages. The last two lines require that server and client have not been corrupted.

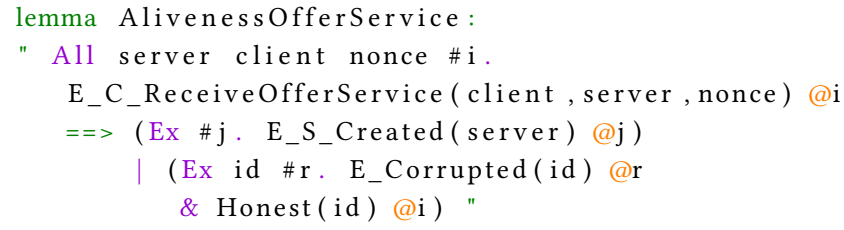

The property fails because the attacker can easily forge messages from an arbitrary Service ID. There is no mechanism in SOME/IP that binds the Service ID within an OfferService message to its sender. The same holds for the Client ID that is part of service requests. The attacker can use any Client ID when sending a request from a compromised ECU. Combining these two weaknesses, the attacker can conduct a MITM attack which requires minimal a priori knowledge of the system. In the following, we describe these practical MITM attacks we derived from our formal analysis.

\subsection{Copycat Attack on the Service Offer}

The general idea of the copycat attack is waiting for a regular server $S$ to send a broadcast service offer and then immediately send a service offer but with the endpoint option of the attacker $A$.

Figure 2 illustrates the attack with an example. First, the regular $S$ sends the service offer message OfferService( $0 \times 1234,0 \times 5678)$ with Service ID 0x1234 and Instance ID 0x5678 and endpoint option Endpoint (10.0.0.2:30509). As soon as $A$ receives this message, she sends the same service offer but with her own endpoint option Endpoint 10.0.0.4:30510. A client $C$ would (usually) receive both service offers, since the attacker is not capable to influence lower-layer communication. $C$ may choose $A$ as the service provider to send his requests to. $A$ can now forward these requests to the original server $S$ as well as the responses from $S$ back to $C$. In this MITM position, $A$ can perform further attacks, e.g., manipulating message contents or (selectively) dropping messages.

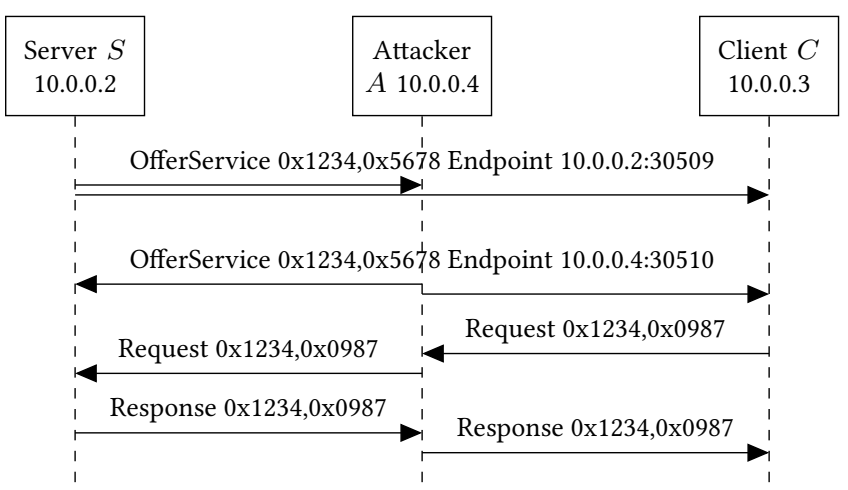

Figure 2: Copycat Attack on the Service Offer

In the Tamarin model, the presence of this attack can be shown by verifying that whenever a client receives a response from a server which contains some random data that was newly generated by the server and, thus, unknown by the attacker before the send event, the response was sent by the server to the correct client endpoint. For this lemma, Tamarin produces an attack trace that corresponds to the described attack.

In practice, the copycat attack has several problems (from the point of view of the attacker). First, a client may have already established a connection with the server and will not change to another server. Second, even if this is not the case, there is no guarantee that the client will choose the attacker as the service provider. Since the service offer of the original server is always sent before the attacker's service offer, the client may never choose the attacker as a service provider. This approach is also in conflict with the SOME/IP specification, which demands that SOME/IP service instances are unique. Thus, this might enable the detection of the attack with an Intrusion Detection System (IDS). However, as our 
implementation and evaluation have shown (cf. Section 6), a regular server simply ignores such messages.

Alternatively, the attacker could use a different Instance ID. A client, who accepts other service instances, could possibly choose the attacker as before. However, there is still no guarantee that the client chooses the attacker as service instance. In addition, the attacker needs to know valid Instance IDs the client accepts (if the client does not accept all Instance IDs).

In case the load balancing options are used (cf. Section 3), the attacker may also send a service offer with the highest priority and weight option. If other instances with the same priority and weight are present, the attacker still has a chance to be chosen randomly.

\subsection{De-association Attack on the Service Offer}

To address the aforementioned issues, we extend the copycat attack by making the clients $C$ believe that the service of the regular server $S$ is no longer available. For this, $A$ additionally de-associates $C$ from $S$ by sending a unicast Stop0ffer message with the regular server's endpoint option.

Figure 3 shows an exemplary attack. $S$ sends 0 fferService( 0x1234, 0x5678) with Endpoint (10.0.0.2:30509). Then, $A$ immediately sends Stop0ffer $(0 \times 1234,0 \times 5678)$ for Endpoint (10. $0.0 .2: 30509$ ) of $S$ as unicast to $C$ with interest in the service. As in the copycat attack, $A$ also immediately sends her own service offer to get in the MITM position.

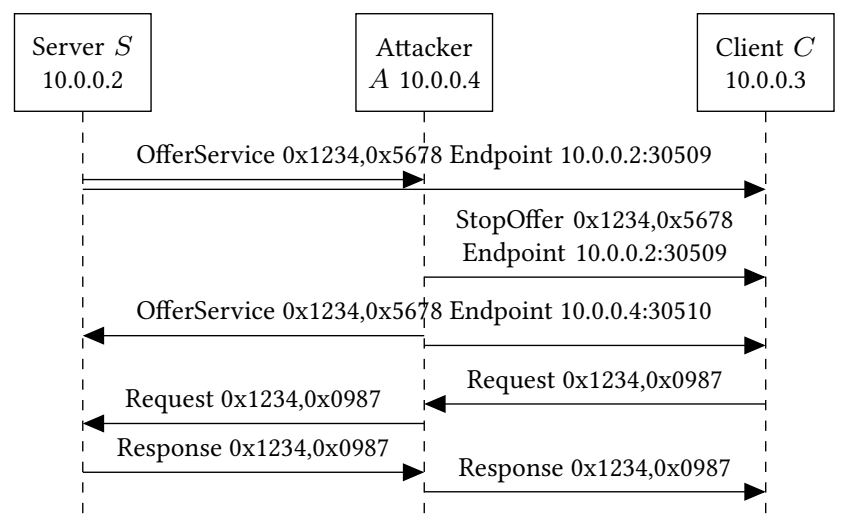

Figure 3: De-association Attack on the Service Offer

Tamarin generates this attack, if the lemma mentioned in the previous section is further restricted to cases where the server was known by the client a priori.

The StopOffer messages are sent to the clients as unicast, since if $S$ would receive this, it would stop providing the service, assuming another server would take over. This would prevent $A$ from getting into the MITM position. The OfferService message of the attacker can be sent as broadcast since $S$ just ignores this message.

The de-association attack has the drawback that $A$ needs to know the clients which are interested in the service. Otherwise, she could not send unicast StopOffer messages to them. Thus, the attacker needs to identify these clients. Since $A$ can only eavesdrop on broadcast communication, she can either listen to broadcast FindService() messages of clients or other broadcast messages which identify clients and their IP addresses. Alternatively, $A$ can simply send the StopOffer message to the entire IP range of the network (excluding the regular server).

\subsection{Attack on Publish/Subscribe}

Based on the request/response attack discovered with Tamarin, we developed an attack on publish/subscribe as shown in Figure 4. The regular server sends OfferService $(0 \times 1234,0 \times 5678)$ with Endpoint (10.0.0.2:30509). Then, $C$ and $S$ subscribe to the service with SubscribeEventgroup $(0 \times 1234,0 \times 5678)$ and their respective endpoint option, i.e., Endpoint (10.0.0.3:3333) for $C$ and Endpoint (10.0.0.4:4444) for the attacker. $S$ acknowledges this by sending SubscribeEventgroupACK $(0 \times 1234,0 \times 5678)$.

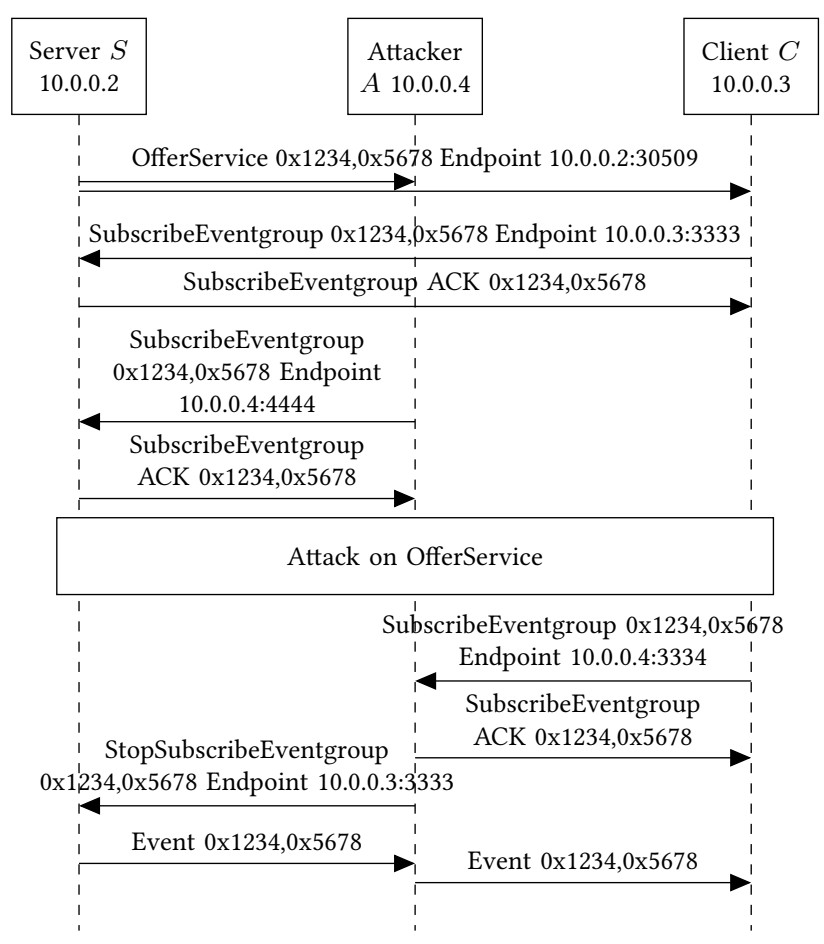

Figure 4: Attack on Publish/Subscribe

$A$ also performs - as fast as possible - one of the presented attack on the service offer, making $C$ believe that the attacker offers the desired service. $C$ sends SubscribeEventgroup $(0 \times 1234$, 0x5678) with Endpoint (10.0.0.4:3334) to subscribe to $A$. $A$ sends StopSubscribeEventgroup $(0 \times 1234,0 \times 5678)$ with $C$ 's endpoint option Endpoint (10.0.0.3:3333) to $S$ for unsubscribing $C$ and stopping $S$ to send event messages to $C$. A sends the Acknowledgment SubscribeEventgroupACK $(0 \times 1234,0 \times 5678)$ to $C$.

$C$ can now perform MITM attacks. When $S$ sends event messages Event $(0 \times 1234,0 \times 5678)$ for his service, these messages are sent to the attacker and not to $C$. $A$ can then decide what to do with these messages, e.g., alter them before forwarding them to the client or drop them. 


\section{ATTACK EVALUATION}

In this section, we briefly describe our implementation of the attacks with vsomeip and CANoe as well as the evaluation.

\subsection{Attack Implementation}

Our first implementation consists of a client, a server offering a service, and the MITM attacker which are each implemented on an ARM1176JZF-S board running Linux Debian (Kernel 4.19) and vsomeip [7] 3.1.16.1. The three boards are connected via a switch and communicate via UDP (or TCP with similar results). We used the examples shipped with vsomeip as test cases for request/response and publish/subscribe communication. For request/response, the server offers a service for around 10 seconds and regularly sends OfferService messages (at the latest every 2.5 seconds). After 10 seconds, the server sends a Stop0ffer message and waits for around 10 seconds before it starts over. The client requests data around every second while the service is offered. Note that there are small variations when messages are sent depending on the load of the system and network. The publish/subscribe example starts the same way. Instead of a request, the client sends a subscribe message as soon as the service is available which is acknowledged by the server. The server then sends events every second to the subscribed client. The attacker is implemented with Scapy [6].

In our second implementation, SOME/IP client and server are implemented on a PC running CANoe 9.0.137 [11] with the SOME/IP library in combination with the VN5610 Automotive Ethernet interface. PC and VN5610 are connected via a switch. The attacker is implemented in a Linux VM on the PC. The SOME/IP communication is implemented in the same way as in the vsomeip implementation.

\subsection{Evaluation of Service Offer Attacks}

The copycat and de-association attack on the service offer (cf. Section 5.1 and 5.2) have the goal to route messages via the attacker. For our evaluation, we run the request/response example for 1000s and evaluate the number of response messages, which are sent directly from server to client and the messages sent via the attacker.

Table 1 shows the results of our evaluation with vsomeip and CANoe. For both, we compare the three cases: no attack, copycat attack, and de-association attack. The column "Direct" shows the number of response messages sent directly from the regular server to the client, i.e., for these messages, our attack was not successful. The column MITM shows the number of response messages sent via the MITM attacker, i.e., the attack was successful and these messages are redirected to our MITM attacker.

Table 1: Evaluation of Copycat and De-association Attack

\begin{tabular}{l|cc|cc} 
& \multicolumn{2}{|c|}{ vsomeip } & \multicolumn{2}{c}{ CANoe } \\
& Direct & MITM & Direct & MITM \\
\hline No Attack & 458 & 0 & 498 & 0 \\
Copycat & 471 & 0 & 0 & 497 \\
De-association & 2 & 473 & 0 & 499
\end{tabular}

When implementing our attacks in vsomeip we had the following result: Without attack, in total 458 response messages were sent, i.e., during the 10 second sending period 9 to 10 messages have been sent. As expected, all of these messages are sent directly from the regular server to the client.

In the case of the copycat attack, in total 471 messages were sent and all of them were sent directly. The implementation only accepts new services for the same Service ID with a different IP if the time to life (TTL) of the original service is expired. Thus, the attack was not successful at all. In our example application, this occurs only after a StopOffer message has been sent.

In contrast to the copycat attack, the de-association attack works very well. Only two of the 475 total response messages were sent directly from the server to the client. All other 473 messages were sent via the attacker. The reason for the two missed messages is the non-deterministic timing, i.e., the server's response was sent before the attacker could send a Stop0ffer message.

In comparison to vsomeip, we observed different results for our attacks on the SOME/IP implementation using CANoe. Both, the copycat and the de-association attack are successful in the request/response scenario. All messages are redirected via our MITM attacker. The results indicate the implementation favors the last Service0ffer contrary to vsomeip. Since this implementation is closed source, we cannot verify this observation.

\subsection{Evaluation of Publish/Subscribe Attack}

Attacks on publish/subscribe can be either combined with copycat or de-association attack. Table 2 shows these two cases and the case without attack for our implementations in vsomeip and CANoe.

When implementing our attacks in vsomeip we had the following results. Without attack, the server sends 556 event notifications during the evaluation period of 1000 seconds directly to the client. In total, the server generates event messages for 556 events. For all of the 556 events, the server generates respective event messages and sends them to the attacker. However, the server also generates 380 event messages for some of these events and sends these event messages directly to the client. Thus, the client receives some events twice. The reason is in a different implementation compared to the request/response handling. Clients subscribe to all suitable services as soon as they receive a ServiceOffer. Since the server regularly sends OfferService messages, the client subscribes each time again and the server sends some events to the client since the attacker is not fast enough to send a StopSubscribeEventgroup message in time.

Table 2: Evaluation of the Attack on Publish / Subscribe

\begin{tabular}{l|cc|cc} 
& \multicolumn{2}{|c|}{ vsomeip } & \multicolumn{2}{c}{ CANoe } \\
& Direct & MITM & Direct & MITM \\
\hline No Attack & 556 & 0 & 500 & 0 \\
Copycat & 380 & 556 & $(499)$ & 499 \\
De-association & $0(385)$ & $(0) 422$ & $(500)$ & 500
\end{tabular}

The attack in combination with the de-association attack does not work with the reference implementation of vsomeip, due to some implementation error. Clients ignore the first OfferService after a StopOffer message. We indicate this behavior with $(0)$ in the table. To address this issue, we are sending two OfferService messages after a StopOffer, and the MITM attack works. The server 
sends 422 event messages to the attacker, who forwards them to the client. In addition, the server sends 385 event messages directly to the client, which are rejected since the client has already received the Stop0ffer message for this server. The total number of received event messages at the client is lower than in the first two cases (422 compared to 556) since all attack steps (subscription handshake between attacker and server, subscription handshake between attacker and client, StopSubscribeEventgroup, a StopOffer, and two OfferService messages) require some time before the attacker is in the MITM position.

Using the CANoe implementation of SOME/IP, both attacks are effective in the publish/subscribe case. Contrary to vsomeip, the CANoe implementation server does not stop sending to a client even if the attacker sends a StopSubscribeEventgroup. However, the client ignores these messages after the Stopoffer message.

\section{SECURITY EXTENSIONS}

The presented attacks on SOME/IP-SD at application layer are possible even when security mechanisms are deployed at lower layers. An attacker, who has compromised a single ECU (not necessarily a service offering server) can authenticate as a legitimate member of the network and perform the MITM attacks.

In Section 2, we already discussed related work for securing SOME/IP with an IDS [12], TLS [18], TESLA [18], and digital signatures with asymmetric cryptography [15]. Approaches such as TLS and digital signatures with asymmetric cryptography can achieve a high level of security while meeting general automotive requirements (e.g., zero false positives, low latency). Both approaches can prevent an outsider from performing MITM attacks. However, in our attacker model we also consider an insider attacker who can authenticate and execute MITM attacks. Also, TLS and the approach proposed in [15] cannot be used for SD broadcast communication.

In the next sections, we propose two security extensions for SOME/IP. To prevent or at least mitigate MITM attacks on SOME/IP$\mathrm{SD}$ of an insider, we propose to restrict keys to the services which the ECU is allowed to offer and/or to use. Thus, an attacker who has compromised an ECU and the keys can only offer and use services that are allowed, mitigating the impact of the attacker.

Secure SOME/IP service discovery and session establishment using restricted certificates and digital signatures (SESO-RC) secures SOME/IP-SD broadcast messages and all subsequent SOME/IP communication. Similar to [15], our approach uses initially digital signatures with asymmetric cryptography for establishing symmetric keys for securing the subsequent communication. Certificates contain information about authorized services to limit the possible impact of an ECU compromise. In contrast to [15], our approach still enables SD broadcast communication and thus, does not change the specified SOME/IP protocol flow.

Secure SOME/IP service discovery and session establishment using an authorization server (SESO-AS) uses only efficient symmetric cryptography but requires an additional authorization server (AS) in the E/E architecture. The AS also stores additional information about the authorized services of an ECU. We assume that the AS is especially protected against compromise, e.g., by means of a hardware security module and mechanisms like secure boot.

\subsection{SESO-RC}

Each ECU is equipped with a private secret key $s k_{E C U}$ and corresponding certificate $\operatorname{Cert}_{E C U}$ (including the public key $p k_{E C U}$ ). Certificates are issued by a trusted certification authority $(\mathrm{CA})$ and all ECUs store the CA public key for verifying the validity of certificates of other ECUs. A certificate contains additional information about the services the ECU is allowed to offer, stop, request, or subscribe to. For the sake of simplicity, we assume all (trusted) certificates are preconfigured on all ECUs and the hash of a certificate is used as a unique identifier $I D_{E C U}$ of an ECU. Alternatively, the entire certificate could be sent within SOME/IP messages and ECUs store only a trusted root CA certificate for verifying received certificates. The preconfigured certificates could, for example, be deployed during production and updated via firmware updates. How this can be done securely is out of scope for our paper.

The service offer is signed with the private key of the sender and includes an ephemeral-static ${ }^{2}$ Diffie-Hellman (DH) key exchange to establish a symmetric session key. The session key is subsequently used to protect the integrity of the following SOME/IP messages by appending a Message Authentication Code (MAC). In addition, a timestamp ensures the freshness of messages if ECUs are loosely time-synchronized. Otherwise, a counter could be used.

Figure 5 illustrates SESO-RC for a request/response communication. The server $S$ stores a certificate Cert $_{S}$ containing the public key $p k_{S}$ and a list of services the server is allowed to offer and stop. It also stores the corresponding private key $s k_{S}$ and the public keys of all clients. A client stores a certificate $\operatorname{Cert}_{C}$ containing the public key $p k_{C}$ and a list of services the client is allowed to request. It also stores the corresponding private key $s k_{C}$ and the certificates of all servers (including the respective public keys).

The server broadcasts a service offer message $O S$ including the usual payload such as service ID, instance ID (denoted with ...), identifier $I D_{S}$, a freshness value $f_{1}$, and a DH public key $p k_{S D H-S}$ (used by all clients later in the generation of the shared symmetric keys). This message is signed with the private key $s k_{S}$ of the server. A client, which wants to request a service, first verifies whether the signature is valid and $f_{1}$ is current. If the verification passes, the client generates an ephemeral DH key pair and includes the public part $p k_{E D H-C}$, a freshness value $f_{2}$ dependent on $f_{1}$, and $I D_{C}$ in the service request message Req, signed with the private key $s k_{C}$ of the client. Client and server can now both calculate the shared symmetric key $k_{S C}$ with their private DH key and the received public key. Other clients also use $p k_{S D H-S}$ of the server but generate their own ephemeral $\mathrm{DH}$ key pair so that each connection uses an individual symmetric key. Finally, the server sends the response message Res including the requested service data, $I D_{S}$ and a freshness value $f_{3}$. Since client and server now possess the shared symmetric key $k_{S C}$, this and further message are secured with a MAC.

Figure 6 illustrates SESO-RC for Publish/Subscribe. Since the server later broadcasts events to all clients $C_{i}$ (where $i$ is used for numbering of the clients) which are subscribed to the service, a group key is required. The first two steps for the service offer $O S$ and Subscribe Eventgroup $S E$ messages are similar as for the request/response. All clients $C_{i}$ have established individual symmetric

\footnotetext{
${ }^{2}$ only static for one service offer as all clients use the same server $\mathrm{DH}$ public key
} 


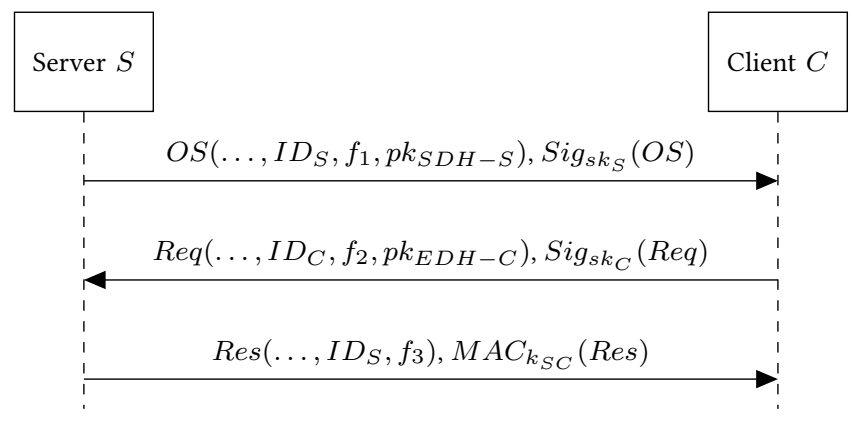

Figure 5: SESO-RC with Request/Response

keys $k_{S C_{i}}$. The server generates a group key $k_{\text {group }}$ which is individually encrypted for each client with $k_{S C_{i}}$ and distributed in the Subscribe Eventgroup Acknowledgment message $S E A$. To prevent replay attacks, the encryption contains a freshness value. A MAC is calculated using $k_{S C i}$ and appended to ensure integrity. Note that we use $k_{S C_{i}}$ for both encryption and MAC only for the sake of simplicity. Since one should use distinct keys for each purpose, $K_{S C_{i}}$ should be used only as a basis for deriving distinct keys.

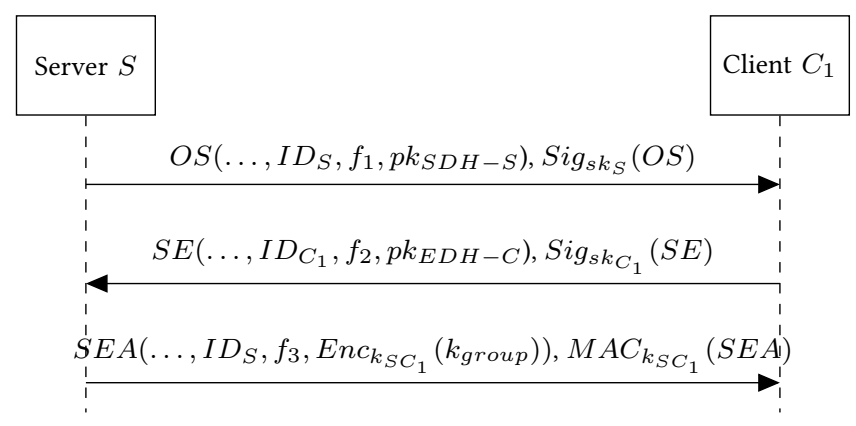

Figure 6: SESO-RC with Publish/Subscribe

\subsection{SESO-AS}

Each ECU is assigned with a unique identifier $I D_{E C U}$ and symmetric key $k_{E C U}$. Again, we assume that these values are preconfigured and stored on the ECU as well as at the AS. For each ECU, the AS also stores additional information about the services the ECU is allowed to offer, stop, request, or subscribe to. Similar to SESO-RC, some mechanism to ensure freshness is deployed. Thus, either all ECUs and AS are loosely time-synchronized or a counter is used.

Figure 7 illustrates SESO-AS for the Offer Service. A server sends a service offer $O S$, including the usual payload such (denoted with $\ldots), I D_{S}$, a freshness value $f_{0}$, the encrypted session key $k_{s e}$ and a MAC to the AS. The AS verifies MAC, $f_{0}$, and whether the server is allowed to offer this service. If the verification passes, the AS derives shared symmetric keys $k_{S C_{i}}$ from $k_{s e}$ for the server and clients $C_{i}$ which are authorized to use (request or subscribe) this service. These keys are individually encrypted for each $C_{i}$ using $k_{C_{i}}$. Then the AS broadcasts the service offer message $O S$ with the usual payload and a new freshness value $f_{1}$. The AS appends individual containers $E_{i}$ for each legitimate client $C_{i}$ to the message. For example, $E_{1}$ contains $I D_{C_{1}}$, the encrypted symmetric key $\left.E n c_{k_{C_{1}}}\left(k_{S C_{1}}\right)\right)$ and a MAC $M A C_{k_{C_{1}}}\left(O S, E_{1}\right)$. Client $C_{1}$ verifies the MAC, decrypts $k_{S C_{1}}$, and uses $k_{S C_{1}}$ to secure subsequent messages with a MAC.

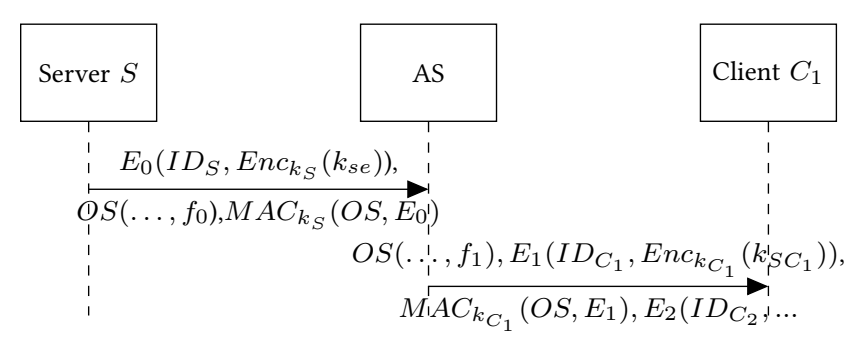

Figure 7: Offer Service with SESO-AS

\subsection{Security Analysis and Verification}

We use the Tamarin prover to verify the key exchange during SOME/IP-SD of our proposed protection mechanisms. The corresponding Tamarin models are given in Appendix A.2 and A.3. In contrast to our analysis of SOME/IP, we do not assume underlying security protocols but allow the attacker full control over the network. We abstract the freshness values $f_{n}$ with random nonces.

7.3.1 SESO-RC. For SESO-RC we verify strong authentication of session keys as well as forward-secrecy of session keys with the following two security properties (cf. Section 4.2):

Definition 7.1 (Injective-agreement on session keys). For an honest server $S$ and honest client $C$ it holds that whenever $S$ establishes a session, apparently with $C$, then $C$ has previously established a session, apparently with $S$, and $C$ and $S$ agree on all parameters of the session, especially the session key. Moreover, each session established by $S$ corresponds to a unique session established by $C$.

Injective agreement not only implies aliveness (cf. Section 5), but also protection against replay and MITM attacks, given that no ECU is authorized to offer and consume a service at the same time.

Definition 7.2 (Forward-secrecy of session keys). For an honest server $S$ and honest client $C$ and a session key $k$ that either of the two parties believes to be a session key shared with the other party, the attacker cannot learn $k$ even if she compromises private keys of $S$ or $C$ later.

7.3.2 SESO-AS. For the formal verification of SESO-AS we assume that access control is done by the authorization server $A S$, that is, $A S$ will only allow key exchanges for a specific service between authorized servers/clients. Therefore, we do not represent this within our model. We require injective agreement on the shared keys between a client and a server $k_{S C_{n}}$ as well as secrecy of the session key $k_{s e}$ and $k_{S C_{n}}$. In contrast to SESO-RC, SESO-AS does not provide perfect forward-secrecy of session keys as encrypted session keys are transmitted. Both properties can be verified in Tamarin.

Definition 7.3 (Injective agreement on shared keys). For an honest server $S$, honest client $C$, and honest authorization server $A S$, it holds that whenever $C$ receives an OfferService message, apparently from $S$, then $S$ has sent an offerService message before and 
the shared symmetric key $k_{S C}$ received by $C$ has been correctly derived from the session key $k_{s e}$. Moreover, each received event by $C$ corresponds to a unique send event by $S$.

Definition 7.4 (Secrecy of shared keys). For an honest server $S$, honest authorization server $A S$, and every session key $k_{s e}$ generated by $S$, the attacker cannot learn $k_{s e}$.

In addition, for an honest server $S$, honest client $C$, and honest authorization server $A S$, it holds that whenever $C$ receives an OfferService message with a shared symmetric key $k_{S C}$, apparently shared with $S$, the attacker cannot learn $k_{S C}$.

\subsection{Performance Evaluation}

In this section, we describe the implementation and evaluation of the performance overhead of SESO-RC and SESO-AS and compare them with "Secure SOME/IP" [15]. We excluded [18] from the comparison since service discovery is not protected in this approach. First, we discuss the communication overhead introduced by the increased message size. Then, we analyze the computational overhead by implementing all approaches and measuring the additional latency. Finally, we summarize the performance evaluation of all three approaches.

7.4.1 Message Overhead. SESO-RC adds a freshness value $f$ of 4 bytes to every message. The OfferService() message of the server contains the public key of the elliptic curve Diffie-Hellman (ECDH) key exchange (X25519) with a size of 32 bytes and the SHA-256 hash value of the certificate with a size of 32 bytes. The Ed25519 elliptic-curve signature of the message has a size of 64 bytes. Figure 8 shows the complete message format of the service offer. Request() and SubscribeEventgroup() messages of the client have the same message format. All following messages of client and server add only a 32 bytes HMAC-SHA-512/256 (HMAC with SHA- 512 truncated to 256 bits) and the 4 bytes freshness value.

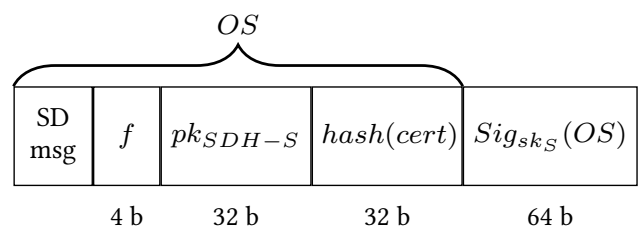

Figure 8: Message Format of Offer Service with SESO-RC

SESO-AS adds a freshness value $f$ of 4 bytes and a set of encrypted keys for each possible client to a OfferService() message. For encryption and authentication, we choose an Authenticated Encryption with Associated Data (AEAD) construction (ChaCha20Poly1305) for improved performance. An entry of the key set consists of a 1 byte identifier of the client $I D_{C_{i}}$, the encrypted key $k_{S C_{i}}$ for a client of 32 bytes, and the AEAD authentication tag of 16 bytes. This authentication tag includes the SOME/IP OfferService message through associated data. The freshness value is used as input nonce for ChaCha20-Poly1305 to encrypt the key $k_{S C_{i}}$. The complete message format is illustrated in Figure 9. All following messages are authenticated with a 32 bytes HMAC-SHA-512/256 and include a 4 bytes freshness value.

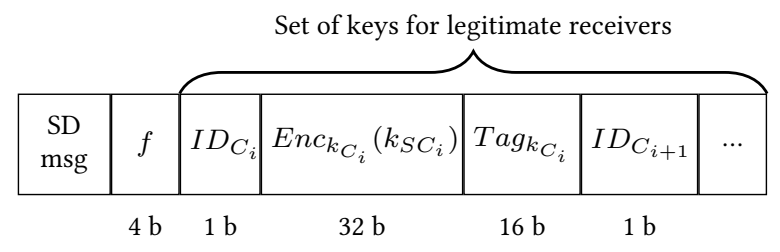

Figure 9: Message Format of Offer Service with SESO-AS

The overhead of regular SOME/IP messages is 36 bytes with both solutions, but they differ in the key exchange handshake depending on the number of clients that request a service. Table 3 shows a comparison of the different approaches for different numbers of clients where msg denotes the number of additional messages and bytes the total value of additional bytes over all necessary messages.

Table 3: Overhead of Cryptographic Key Exchange

\begin{tabular}{ll|ccccc} 
& & \multicolumn{5}{|c}{ Number of Clients } \\
& & 1 & 2 & 4 & 8 & 16 \\
\hline \hline \multirow{2}{*}{ SESO-AS } & msg & 1 & 1 & 1 & 1 & 1 \\
& bytes & 106 & 155 & 253 & 449 & 841 \\
\hline \multirow{2}{*}{ SESO-RC } & msg & 0 & 0 & 0 & 0 & 0 \\
& bytes & 264 & 396 & 660 & 1188 & 2244 \\
\hline \multirow{2}{*}{ "Secure SOME/IP" [15] } & msg & 0 & 2 & 4 & 8 & 16 \\
& bytes & 180 & 360 & 720 & 1440 & 2880
\end{tabular}

SESO-AS introduces a message overhead of 53 bytes to the service offer sent to the AS and an additional offerService message with $4+49 c$ bytes, where $c$ is the number of legitimate clients, which is a broadcast message to every client in the network.

The key exchange of SESO-RC does not introduce additional messages since the service offer message is broadcasted directly to the clients. The service offer and the first request introduce the same overhead of 132 bytes. Every further client requesting the service also adds 132 bytes of overhead.

Secure SOME/IP [15] uses a unicast handshake between every client and the server. To enable a fair comparison, we replaced RSA with Ed25519 EC signatures in their implementation. The client part of the handshake transports 36 bytes of the certificate hash with a freshness value and the server adds 144 bytes since it contains the asymmetrically encrypted and signed group key. Both messages are sent for every additional client.

Comparing the three approaches regarding the number of messages, SESO-RC introduces the least number of additional messages while Secure SOME/IP introduces the most messages since it is not capable of broadcasting service discovery messages. Thus, Secure SOME/IP has the highest overhead in message size as soon as a service is used by more than three clients. The least overhead is introduced by SESO-AS.

7.4.2 CPU overhead. For the evaluation of the CPU overhead introduced by our security extensions, we implemented SESO-RC, SESOAS, and Secure SOME/IP on two ARMv6 boards (ARM1176JZF-S at 
$700 \mathrm{Mhz}$ ) connected via a $100 \mathrm{MBit} / \mathrm{s}$ switch. We used libsodium ${ }^{3}$ as cryptography library.

We evaluate the latency of the cryptographic extensions by counting the additional number of CPU instructions using the perf_events of the Linux Kernel. The measurements do not include network transfer time or interpretation of the SOME/IP message. For SESO-RC and Secure SOME/IP we evaluate client and server side and for SESO-AS additionally the AS side. For all three approaches, we perform 1000 measurements for different numbers of clients and calculate the arithmetic mean and the mean absolute deviation (MAD) to determine the error of our measurements. The results are shown in Figure 10.

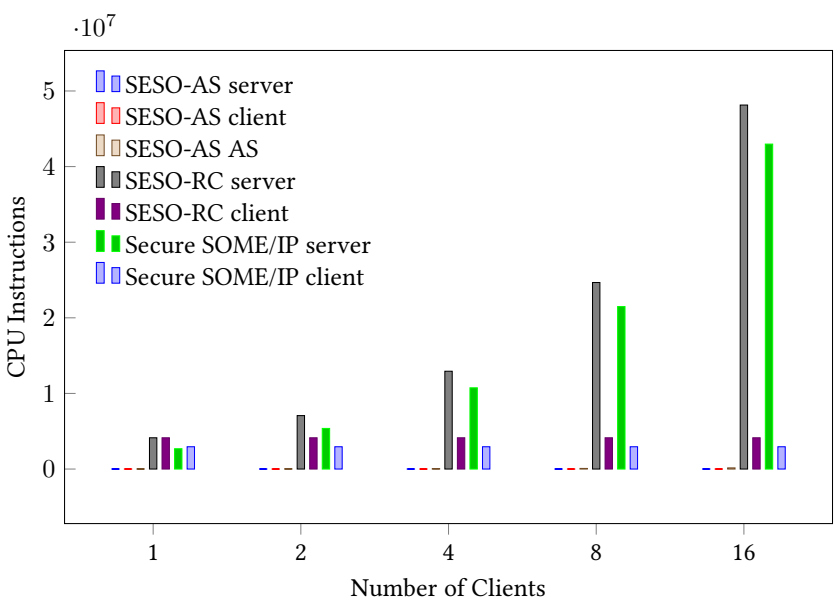

Figure 10: Additional CPU Instructions for Cryptography

SESO-AS clients and servers have a very low overhead of only 7,032 (MAD: 13.1) and 9,182 (MAD: 225.2) instructions independent of the number of clients. The authorization server requires at most 153,937 (MAD: 3,615.6) instructions when encrypting a broadcast message for 16 clients. As expected SESO-AS outperforms SESO-RC which requires 4,134,880 (MAD: 22,059.0) additional instructions for the server and 4,134,550 (MAD: 21,707.0) for the client. Secure SOME/IP in comparison is faster $(2,939,572$ instructions, MAD: 15,371.1) compared to SESO-RC on the client side. The signature verification of every client causes a very high CPU usage of the SESO-RC server compared to the Secure SOME/IP, which does not verify the clients. A larger number of clients increase the CPU usage of SESO-RC and Secure SOME/IP equally compared to a very moderate increase of SESO-AS. Due to the unicast communication for the service offers in Secure SOME/IP, a further delay needs to be considered for the handshake. In an already established system, previously exchanged symmetric keys could be used to validate the client request for a new key, which would significantly improve server performance.

7.4.3 Summary. SESO-AS outperforms the other approaches in terms of latency and message overhead. However, it also introduces a single point of failure in terms of security and safety. Moreover, it is much more complex to introduce new devices into the $\mathrm{E} / \mathrm{E}$

\footnotetext{
${ }^{3}$ https://github.com/jedisct1/libsodium
}

system since the permission list of the authorization server needs to be updated and keys need to be exchanged in advance.

SESO-RC solves these problems with the use of certificates that can be easily distributed, and the distributed authorization can be easily integrated into the SOME/IP protocol. SESO-AS needs a minor protocol change to send all service discovery messages to the authorization server. Both approaches support broadcast service discovery like SOME/IP which Secure SOME/IP does not support. With sufficient processing power, SESO-RC is the best option, since it avoids a single point of failure, provides forward-secrecy and introduces certificates as a simple way to distribute keys and permissions securely. Hardware-based cryptography accelerators may improve the performance for low-performance devices to allow the use of asymmetric cryptography. However, this introduces additional cost. As an alternative, SESO-AS can be used with ECUs with less computational power. However, the protection of the authorization server is of paramount importance for ensuring the security of the whole network.

All approaches protect against our identified MITM attacks. In addition, SESO-RC and SESO-AS provide the ability to create individual secure channels between client and server as well as secure broadcast channels for subscriptions.

\section{CONCLUSION}

Our formal analysis of SOME/IP using Tamarin shows three different types of MITM attacks on SOME/IP even when link layer security mechanisms are deployed. The copycat and de-association attack on the service offer enable an attacker to get in a MITM position for request/response communication. The third attack enables a MITM attack on publish/subscribe using one of the first two attacks. Our implementation and evaluation show the effectiveness of our attacks against two real world SOME/IP libraries. For attacking request/response communication using vsomeip, the de-association attack is the most effective. Nearly all messages are routed via the MITM attacker. Attacking publish/subscribe in combination with the copycat attack using vsomeip enables the redirection of more than $\frac{2}{3}$ of the messages over the attacker. In combination with the slightly adapted de-association attack, all messages the client receives are sent over the attacker. However, in this case some event messages of the server are lost. With CANoe both attacks are successful for request/response and publish/subscribe even though the regular server keeps sending messages to the client in the publish/subscribe case. To protect against these attacks, we present and analyze the security extensions SESO-RC and SESO-AS. Our Tamarin analysis shows the security and our implementation the practical feasibility. SESO-RC is suitable for scenarios where ECUs have sufficient resources for asymmetric cryptography. In contrast to related work, SESO-RC can be still used with broadcast communication for service discovery. In case ECUs have scarce resource constraints, SESO-AS might be a viable solution since it uses only efficient symmetric cryptography but on the cost of introducing the AS as an additional component.

\section{ACKNOWLEDGMENTS}

This research work has been partly funded by the German Federal Ministry of Education and Research (BMBF) and the Hessen State 
Ministry for Higher Education, Research and the Arts within their joint support of the National Research Center for Applied Cybersecurity ATHENE and by the BMBF project VITAF (ID 16KIS0835). Additionally, the project leading to this application has received funding from the European Union's Horizon 2020 research and innovation programme under grant agreement No 883135 .

\section{REFERENCES}

[1] AUTOSAR. 2017. Specification of Module Secure Onboard Communication - CP Release 4.2.2.

[2] AUTOSAR. 2018. Specification of Service Discovery - CP Release 4.2.2. https://www.autosar.org/fileadmin/Releases_TEMP/Classic_Platform_4.4.0/ Communication.zip

[3] AUTOSAR. 2018. Specification on SOME/IP Transport Protocol-CP Release 4.4.0. https://www.autosar.org/fileadmin/Releases_TEMP/Classic_Platform_ 4.4.0/Communication.zip

[4] D. Basin, C. Cremers, J. Dreier, S. Meier, R. Sasse, and B. Schmidt. 2020. Tamarin Prover. Retrieved 2020-09-01 from https://tamarin-prover.github.io/

[5] Karthikeyan Bhargavan, Bruno Blanchet, and Nadim Kobeissi. 2017. Verified Models and Reference Implementations for the TLS 1.3 Standard Candidate. In 2017 IEEE Symposium on Security and Privacy (SP). IEEE, 483-502. https: //doi.org/10.1109/SP.2017.26

[6] P. Biondi and the Scapy community. 2020. Scapy for python3 2.4.3rc1. https: //github.com/secdev/scapy.

[7] BMW AG. 2018. vsomeip 2.10.21. https://github.com/GENIVI/vsomeip.

[8] Z. Cai, A. Wang, W. Zhang, M. Gruffke, and H. Schweppe. 2019. 0-days \& Mitigations: Roadways to Exploit and Secure Connected BMW Cars. Black Hat USA (2019)

[9] Stephen Checkoway, Damon McCoy, Brian Kantor, Danny Anderson, Hovav Shacham, Stefan Savage, Karl Koscher, Alexei Czeskis, Franziska Roesner, and Tadayoshi Kohno. 2011. Comprehensive Experimental Analyses of Automotive Attack Surfaces. In Proceedings of the 20th USENIX Conference on Security (San Francisco, CA) (SEC'11). USENIX Association, USA, 6.

[10] Cas Cremers, Marko Horvat, Jonathan Hoyland, Sam Scott, and Thyla van der Merwe. 2017. A Comprehensive Symbolic Analysis of TLS 1.3 (CCS '17). Association for Computing Machinery, New York, NY, USA, 1773-1788. https: //doi.org/10.1145/3133956.3134063

[11] Vector Informatik GmbH. 2019. CANoe. https://www.vector.com/int/en/products/ products-a-z/software/canoe/

[12] Nadine Herold, Stephan-A. Posselt, Oliver Hanka, and Georg Carle. 2016. Anomaly detection for SOME/IP using complex event processing. In NOMS 2016 - 2016 IEEE/IFIP Network Operations and Management Symposium. IEEE, 1221-1226. https://doi.org/10.1109/NOMS.2016.7502991

[13] IEEE Computer Socienty. 2006. Media Access Control (MAC) Security. IEEE Std 802.1AE-2006.

[14] IEEE Computer Socienty. 2011. Media Access Control (MAC) Security Amendment 1: GCM-AES-256 Cipher Suite. IEEE Std 802.1AEbn-2011.

[15] M. Iorio, A. Buttiglieri, M. Reineri, F. Risso, R. Sisto, and F. Valenza. 2020. Protecting In-Vehicle Services: Security-Enabled SOME/IP Middleware. IEEE Vehicular Technology Magazine (2020).

[16] D. Keuper and T. Alkemade. 2018. The connected car ways to get unauthorized access and potential implications. Computest, Zoetermeer, The Netherlands, Tech. $\operatorname{Rep}(2018)$.

[17] Karl Koscher, Alexei Czeskis, Franziska Roesner, Shwetak Patel, Tadayoshi Kohno, Stephen Checkoway, Damon McCoy, Brian Kantor, Danny Anderson, Hovav Shacham, and Stefan Savage. 2010. Experimental Security Analysis of a Modern Automobile. In Proceedings of the 2010 IEEE Symposium on Security and Privacy (SP '10). IEEE Computer Society, USA, 447-462. https://doi.org/10.1109/SP.2010.34

[18] J. Kreissl. 2017. Absicherung der SOME/IP Kommunikation bei Adaptive AUTOSAR Master's thesis. Universität Stuttgart.

[19] Timm Lauser, Daniel Zelle, and Christoph Krauß. 2020. Security Analysis of Automotive Protocols. In Computer Science in Cars Symposium (Feldkirchen, Germany) (CSCS '20). Association for Computing Machinery, New York, NY, USA, Article 11, 12 pages. https://doi.org/10.1145/3385958.3430482

[20] Li Li, Jun Pang, Yang Liu, Jun Sun, and Jin Song Dong. 2014. Symbolic Analysis of an Electric Vehicle Charging Protocol (ICECCS '14). IEEE Computer Society, USA, 11-18. https://doi.org/10.1109/ICECCS.2014.11

[21] Gavin Lowe. 1997. A Hierarchy of Authentication Specifications. In Proceedings of the 10th IEEE Workshop on Computer Security Foundations (CSFW'97). IEEE Computer Society, USA, 31.

[22] C. Miller and C. Valasek. 2013. Adventures in automotive networks and control units. Def Con 21 (2013), 260-264.

[23] C. Miller and C. Valasek. 2015. Remote Compromise of an Unaltered Passenger Vehicle. Black Hat USA 2015 (2015), 91.
[24] S. Nie, L. Liu, and Y. Du. 2017. Free-fall: Hacking Tesla from wireless to CAN bus. Briefing, Black Hat USA (2017), 1-16.

[25] A. Perrig and J. D. Tygar. 2003. TESLA Broadcast Authentication. Springer US, Boston, MA, 29-53. https://doi.org/10.1007/978-1-4615-0229-6 3

[26] J. Seyler, N. Navet, and L. Fejoz. 2015. Insights on the Configuration and Performances of SOME/IP Service Discovery. SAE Int. F. Passeng. Cars - Electron. Electr. Syst. 8 (04 2015), 124-129. https://doi.org/10.4271/2015-01-0197

[27] A. Sundaresan, L. Gerard, and M. Kim. 2017. Secure ROS. http://secure-ros.csl. sri.com/.

[28] The Tamarin Team. 2019. Tamarin-Prover Manual - Security Protocol Analysis in the Symbolic Model.

[29] Jorden Whitefield, Liqun Chen, Frank Kargl, Andrew Paverd, Steve Schneider, Helen Treharne, and Stephan Wesemeyer. 2017. Formal Analysis of V2X Revocation Protocols. In Security and Trust Management, Giovanni Livraga and Chris Mitchell (Eds.). Springer International Publishing, Cham, 147-163.

[30] Minrui Yan, Jiahao Li, and Guy Harpak. 2020. Security Research on MercedesBenz: From Hardware to Car Control. Black Hat USA (2020).

[31] Daniel Zelle, Christoph Krauß, Hubert Strauß, and Karsten Schmidt. 2017. On Using TLS to Secure In-Vehicle Networks (ARES '17). Association for Computing Machinery, New York, NY, USA, Article 67, 10 pages. https://doi.org/10.1145/ 3098954.3105824

\section{A APPENDIX}

In the following, we provide the Tamarin models we used to analyze SOME/IP as well as our proposed security extensions SESO-RC and SESO-AS. A digital version of the models can be found at https: //code.fbi.h-da.de/seacop/someip-tamarin. Details on Tamarin's specification language can be found in [28].

\section{A.1 Tamarin model of SOME/IP}

As described in Section 5, our Tamarin model of SOME/IP focuses on the authentication properties of service discovery messages. Thus, to reduce the model's complexity, it does not implement Publish/Subscribe communication. However, the identified weaknesses apply to all communication patterns and, thus, the attack can be extended for Publish/Subscribe communication as shown in Section 5.3. In addition, the session ID counters have been abstracted by nonces.

The underlying security protocol is abstracted by a group-secure channel. Only legitimate entities can send or receive messages on this channel and messages cannot be replayed. This corresponds to an authenticated and encrypted communication. Note, that in many cases, only authentication will be used. Thus, the adversary could read but not manipulate the communication. However, we only consider the most restrictive case as we are looking for practical attacks instead of security verification. Similarly, the channel models point-to-point and broadcast communication instead of a bus architecture, as this is more suitable for Automotive Ethernet.

The first set of security lemmas considers the case that the adversary has access to the network but has not corrupted any legitimate ECU and thus, is prevented from manipulation of the network. Next, we consider the case where the adversary is allowed to corrupt ECUs. The model allows for corruption of a single ECU only to get more practical and simple attacks. The last two lemmas correspond to the copycat and de-association attacks described in Section 5.1 and 5.2.

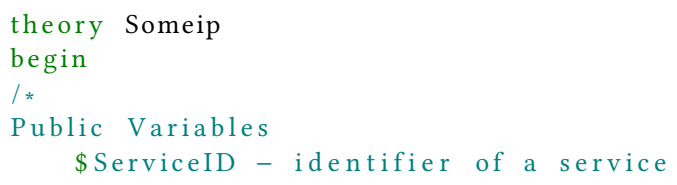



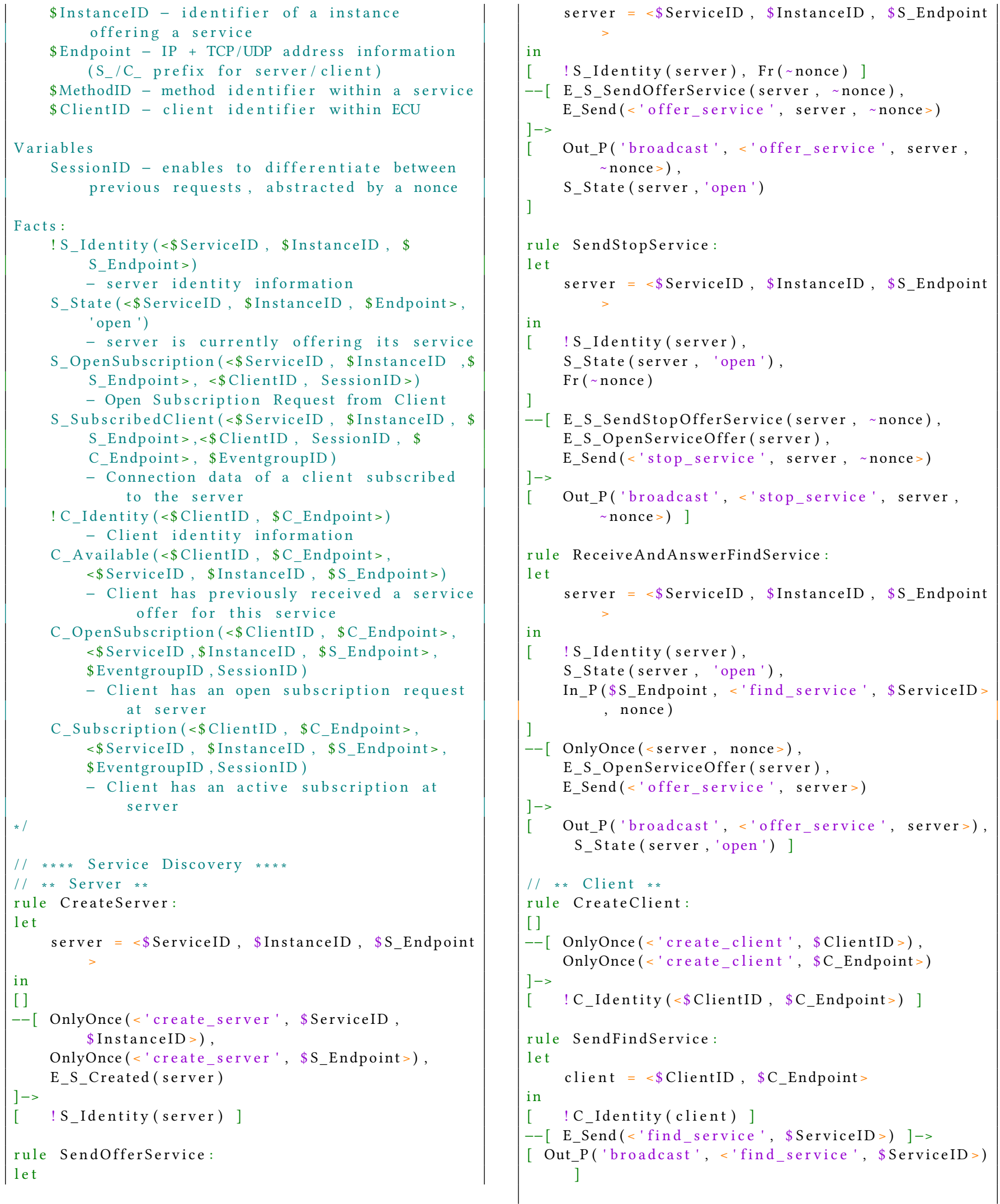


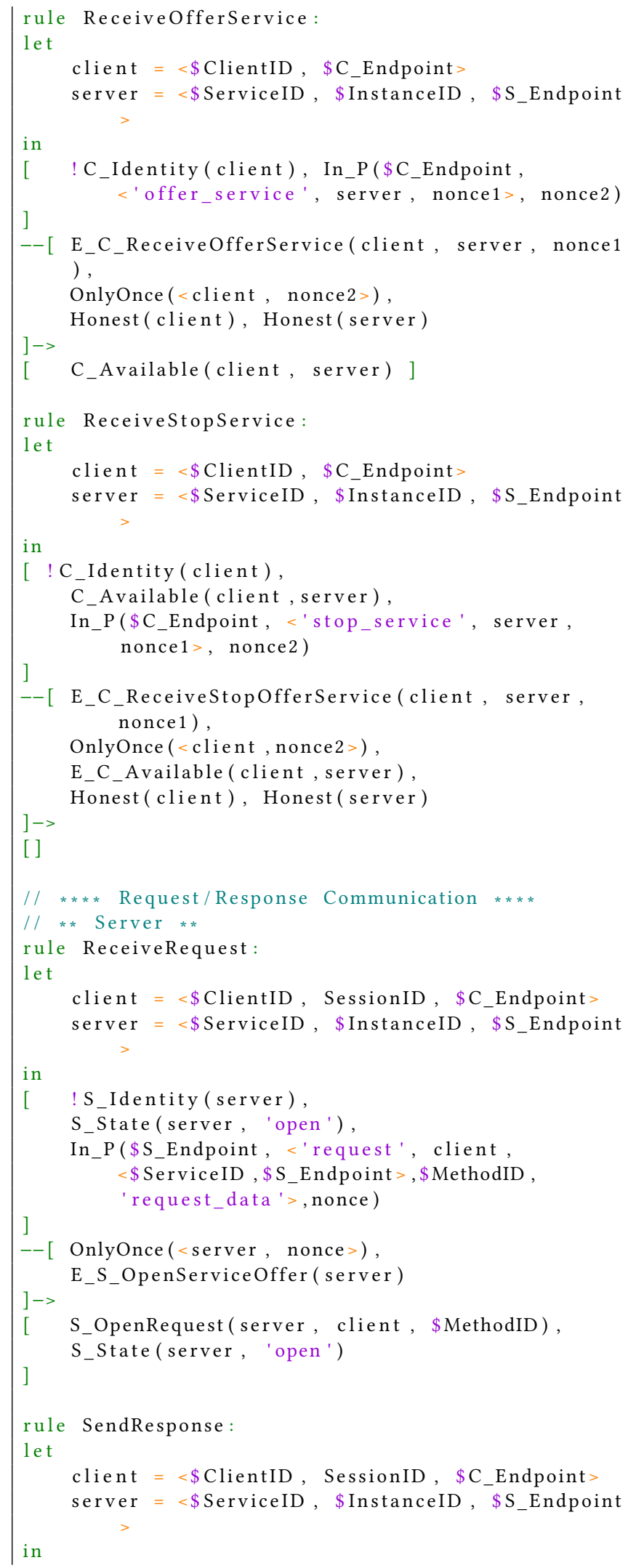

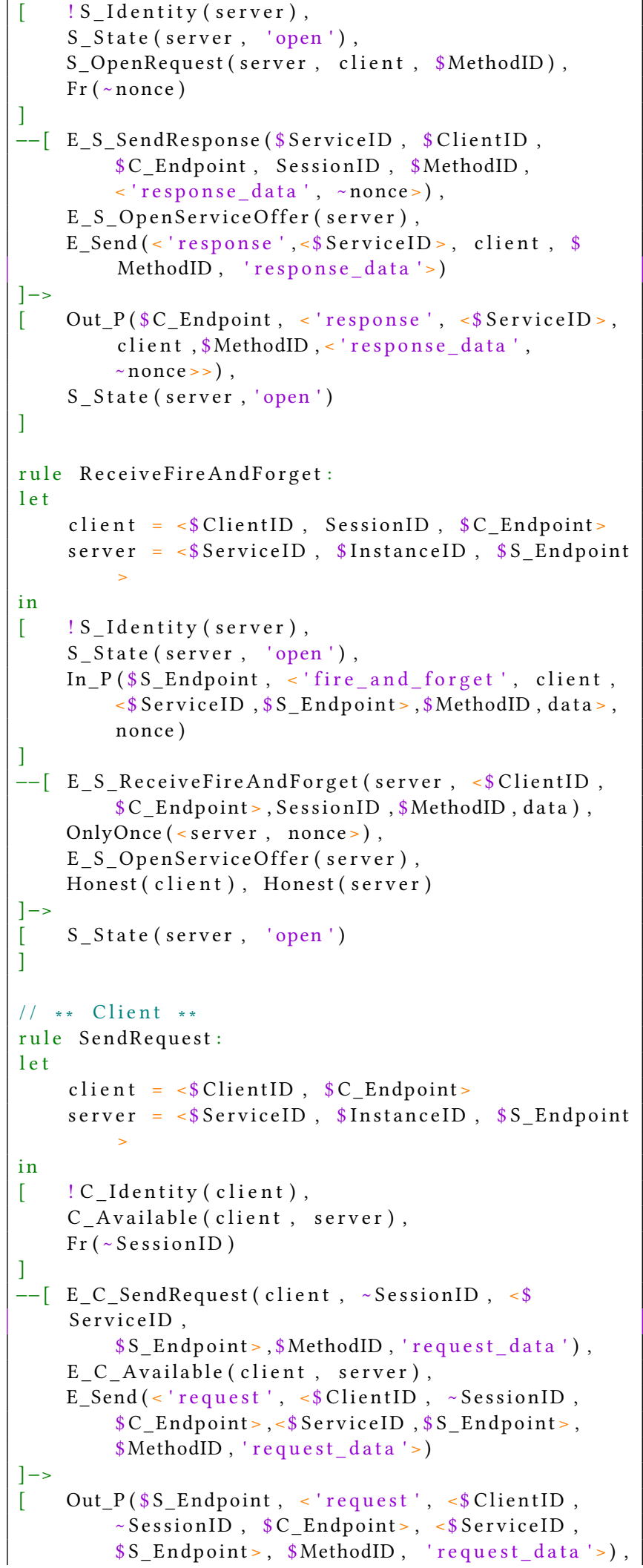




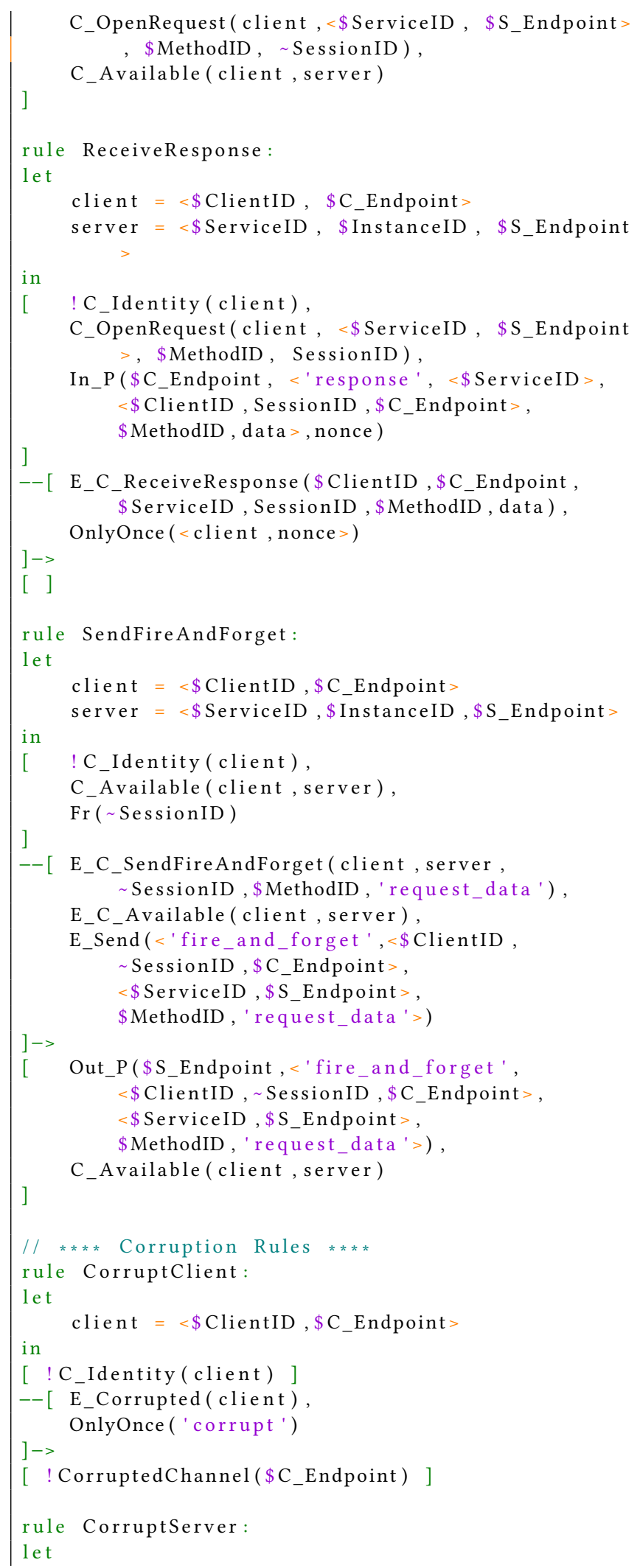

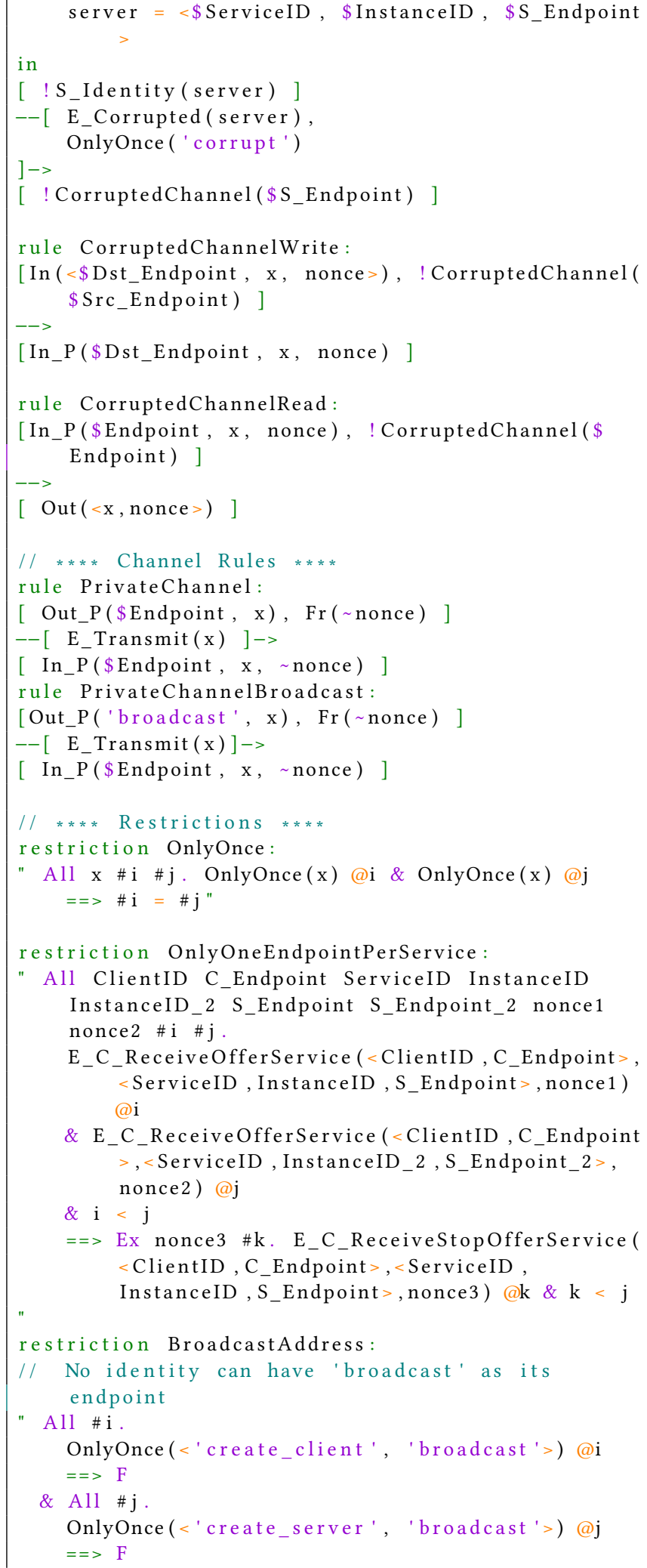




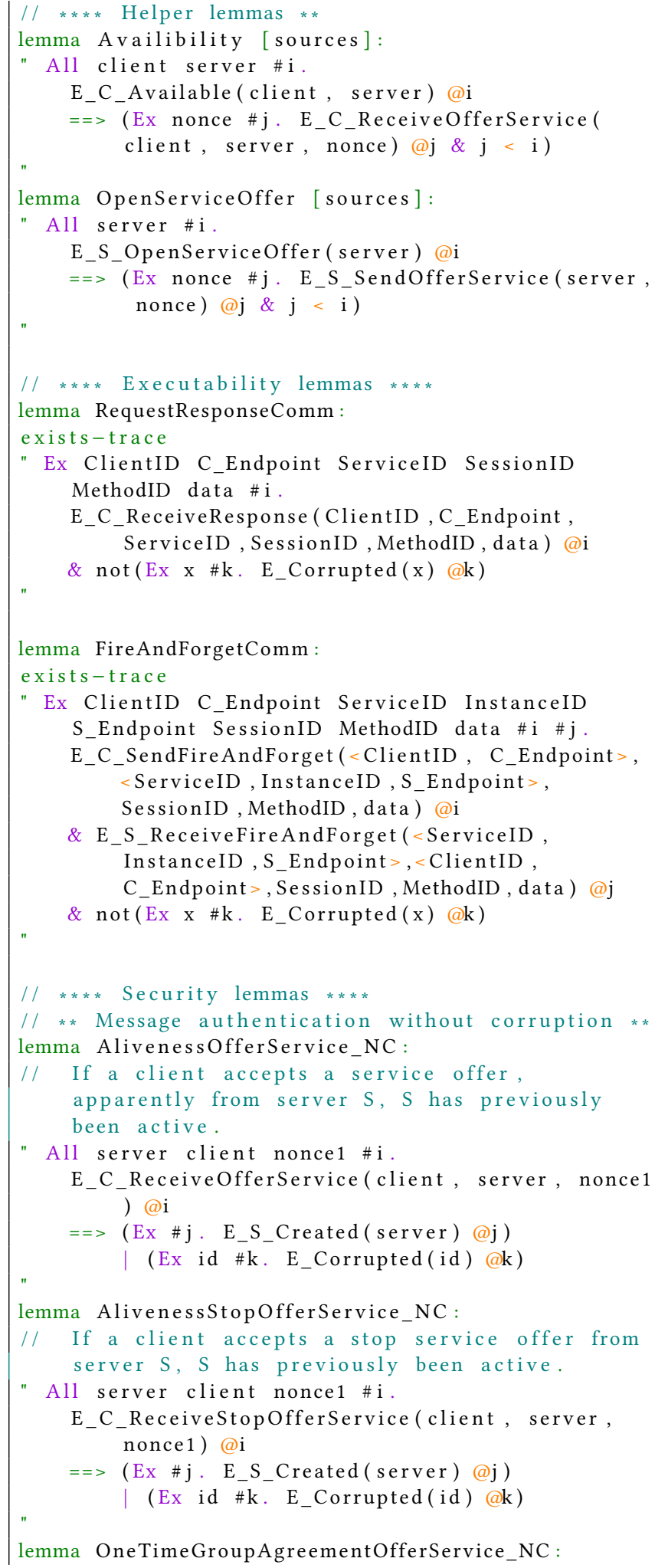

If a client $C$ accepts a service offer from a server $S$, the exact same offer has been previously send by $S$ and has not previously been accepted by $C$.

"All server client nonce \#i.

E_C_ReceiveOfferService(client, server, nonce) $@ \mathrm{i}$

$==>($ Ex \#j. E_S_SendOfferService (server, nonce $)$ @j

$\& \mathrm{j}<\mathrm{i}$

$\&$ not (Ex \#i2. E_C_ReceiveOfferService( client, server, nonce) $@ i 2 \& \operatorname{not}(\# \mathrm{i} 2=\#$ i ) ) )

| (Ex id \#r. E_Corrupted(id)@r)

lemma OneTimeGroupAgreementFireAndForget_NC

// If a server $S$ accepts a fire\&forget message from a client $C$, the exact same message has been previously send by $C$ and has not previously been accepted by $S$.

"All server client session method data \#i. E_S_ReceiveFireAndForget ( server, client, session, method, data)@i

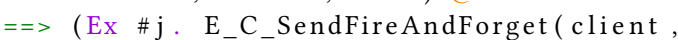
server, session, method, data)@j $\& \mathrm{j}<\mathrm{i}$

$\&$ not (Ex \#i2. E_S_ReceiveFireAndForget ( server, client, session, method, data) @i2 \& $\operatorname{not}(\# \mathrm{i} 2=\# \mathrm{i})))$

| (Ex id \#r. E_Corrupted(id)@r)

$"$

// ** Message authentication with corruption ** lemma AlivenessOfferService:

// If a client accepts a service offer, apparently from server S, S has previously been active.

"All server client nonce \#i

E_C_ReceiveOfferService (client, server, nonce) @i

$==>($ Ex \# j. E_S_Created $($ server $) @ j)$

| (Ex id \#r. E_Corrupted(id)@r \& Honest(id)@ i)

lemma AlivenessStopOfferService:

$/ /$ If a client accepts a stop service offer from server $S, S$ has previously been active.

All server client nonce1 \#i.

E_C_ReceiveStopOfferService(client, server, nonce1)@i

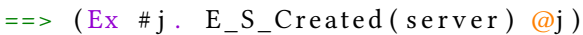

| (Ex id \#r.E_Corrupted(id)@r \& Honest(id)@i )

lemma OneTimeGroupAgreementOfferService:

$/ /$ If a client $C$ accepts a service offer from a server $S$, the exact same offer has been previously send by $S$ and has not previously been accepted by $C$.

"All server client nonce \#i.

E_C_ReceiveOfferService (client, server, nonce) @i 


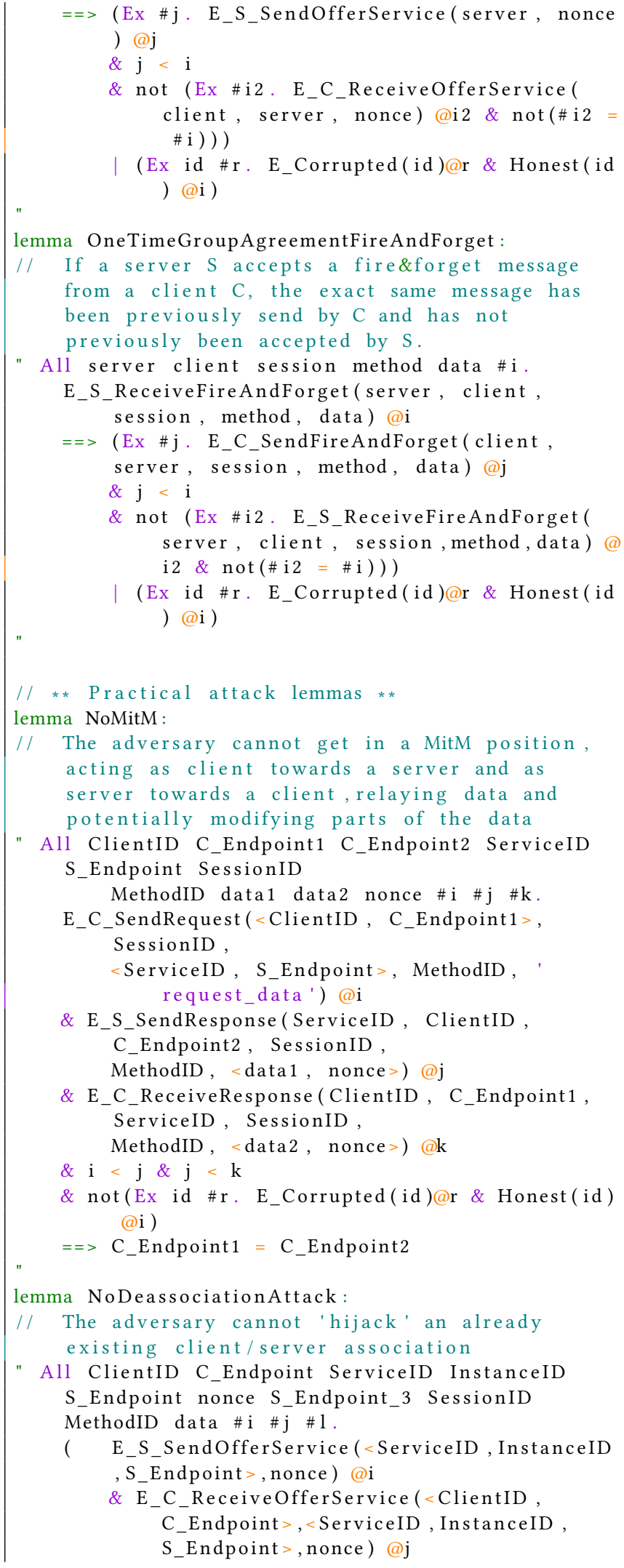

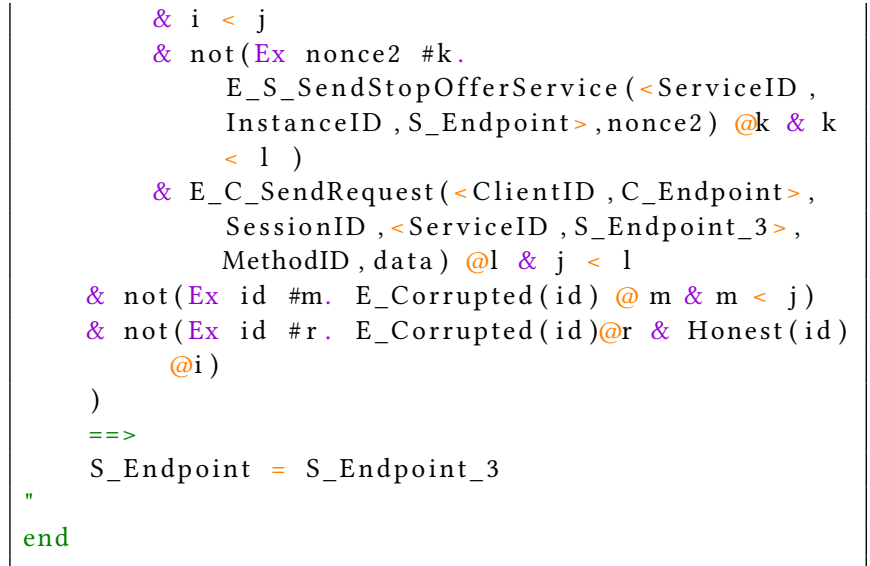

\section{A.2 Tamarin model of SESO-RC}

For our model of SESO-RC (Section 7.1), we focus on the security of the proposed mechanism and, thus, providing a more abstract model than for our SOME/IP evaluation. Freshness values have been abstracted by nonces.

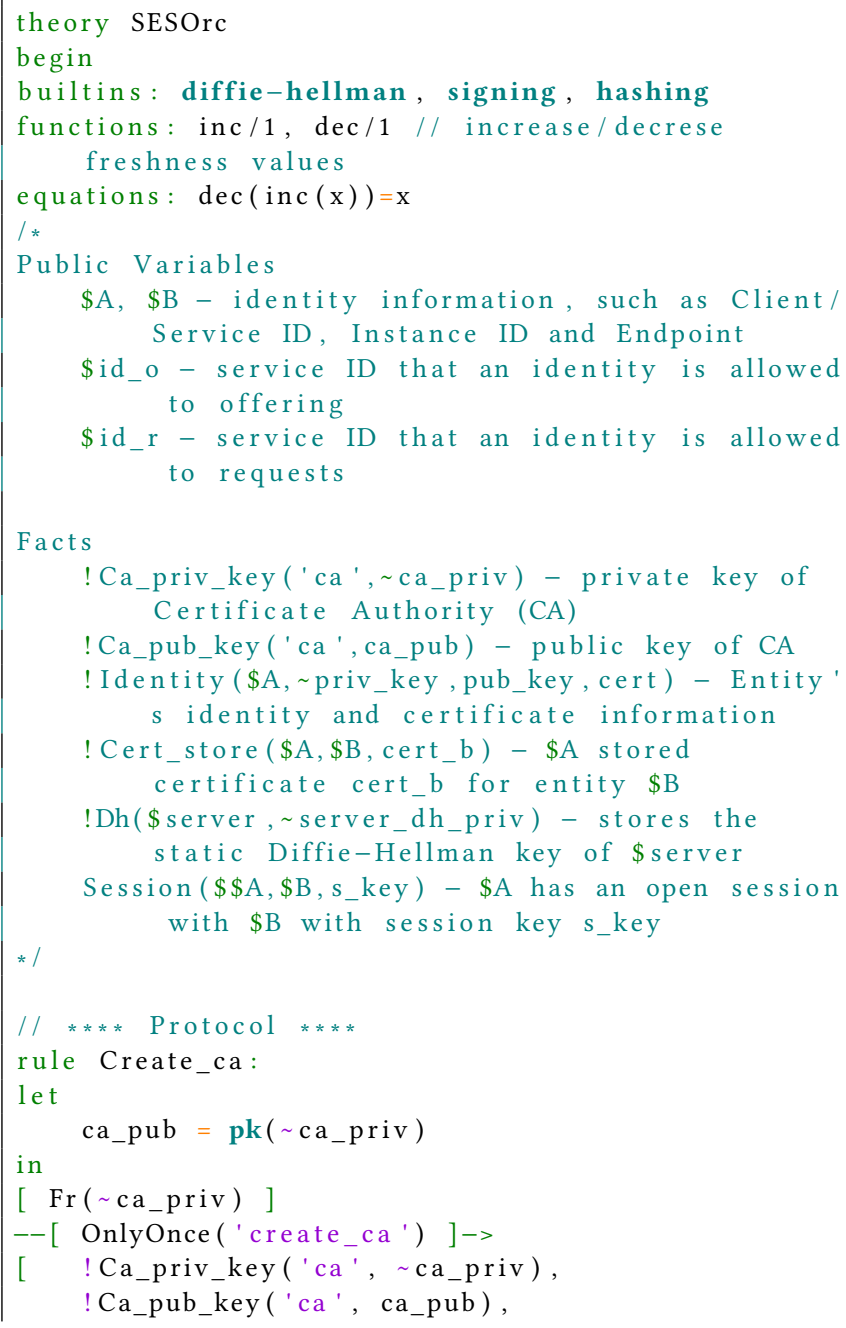




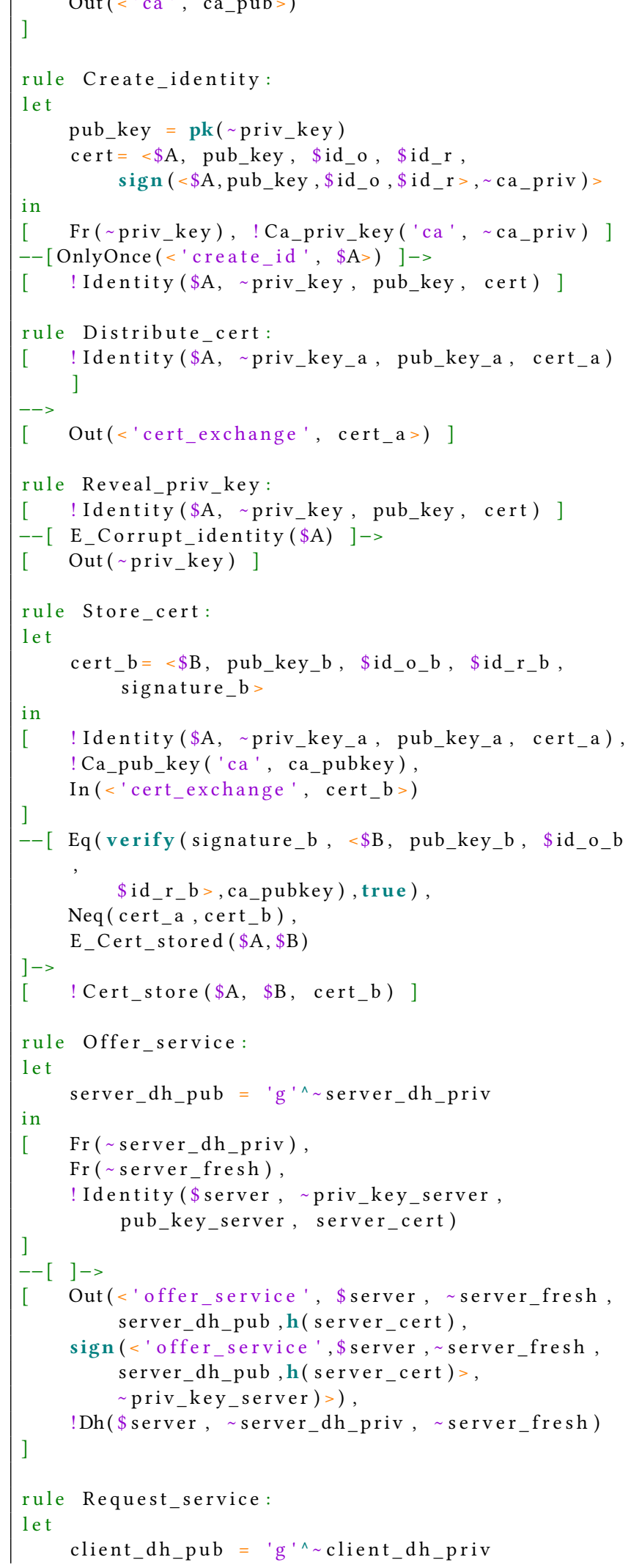

server_cert $=<\$$ server, server_pub_key, \$server_id_o, \$server_id_r, sign_server_cert $>$

client_cert $=<\$$ client, client_pub_key, \$client_id_o, \$client_id_r,

sign_client_cert >

session_key $=$ server_dh_pub^ ${ }^{\wedge}$ client_dh_priv

client_fresh $=$ inc(server_fresh)

in

[ Fr $(\sim$ client_dh_priv $)$,

! Cert_store(\$client, \$server, server_cert),

! Identity (\$client, priv_key_client, pub_key_client, client_cert),

In (<' offer_service', \$ server, server_fresh, server_dh_pub, server_cert_hash, signed_offer $>$ )

]

-- [Eq(verify (signed_offer, <'offer_service', \$server, server_fresh, server_dh_pub, server_cert_hash >, server_pub_key), true),

Eq(h(server_cert), server_cert_hash),

Eq (\$server_id_o, \$client_id_r $)$,

E_C_Session_established(\$client, \$server, client_fresh, session_key),

Honest (\$client), Honest ( \$server),

Secret (session_key)

] $\rightarrow$

[ Session(\$client, \$server, server_dh_pub^ ${ }^{\wedge}$ client_dh_priv),

Out (<'request_service', \$client, \$server, client_fresh, client_dh_pub, h( client_cert),

sign (<'request_service', \$client, \$server, client_fresh, client_dh_pub, $\mathbf{h}($ client_cert $)>, \sim$ priv_key_client $)>$ )

]

rule Response_service_request:

let

server_cert $=<$ server, server_pub_key,

\$server_id_o, \$server_id_r,

sign_server_cert $>$

client_cert $=<\$$ client, client_pub_key, $\$$ client_id_o, \$client_id_r, sign_client_cert >

session_key $=$ client_dh_pub ${ }^{\wedge} \sim$ server_dh_priv

client_fresh $=$ inc $(\sim$ server_fresh $)$

in

[ In (<'request_service', $\$$ client, $\$$ server , client_fresh, client_dh_pub, client_cert_hash, signed_request $>$ ),

! Identity (\$server, priv_key_server, pub_key_server, server_cert),

! Cert_store (\$server, \$client, client_cert),

! Dh( $\$$ server, , server_dh_priv, server_fresh)

]

$--[$ Eq(verify (signed_request, < request_service ', \$client, \$server, client_fresh, client_dh_pub, client_cert_hash >, client_pub_key), true),

Eq(h(client_cert), client_cert_hash), Eq (\$server_id_o, \$client_id_r), 


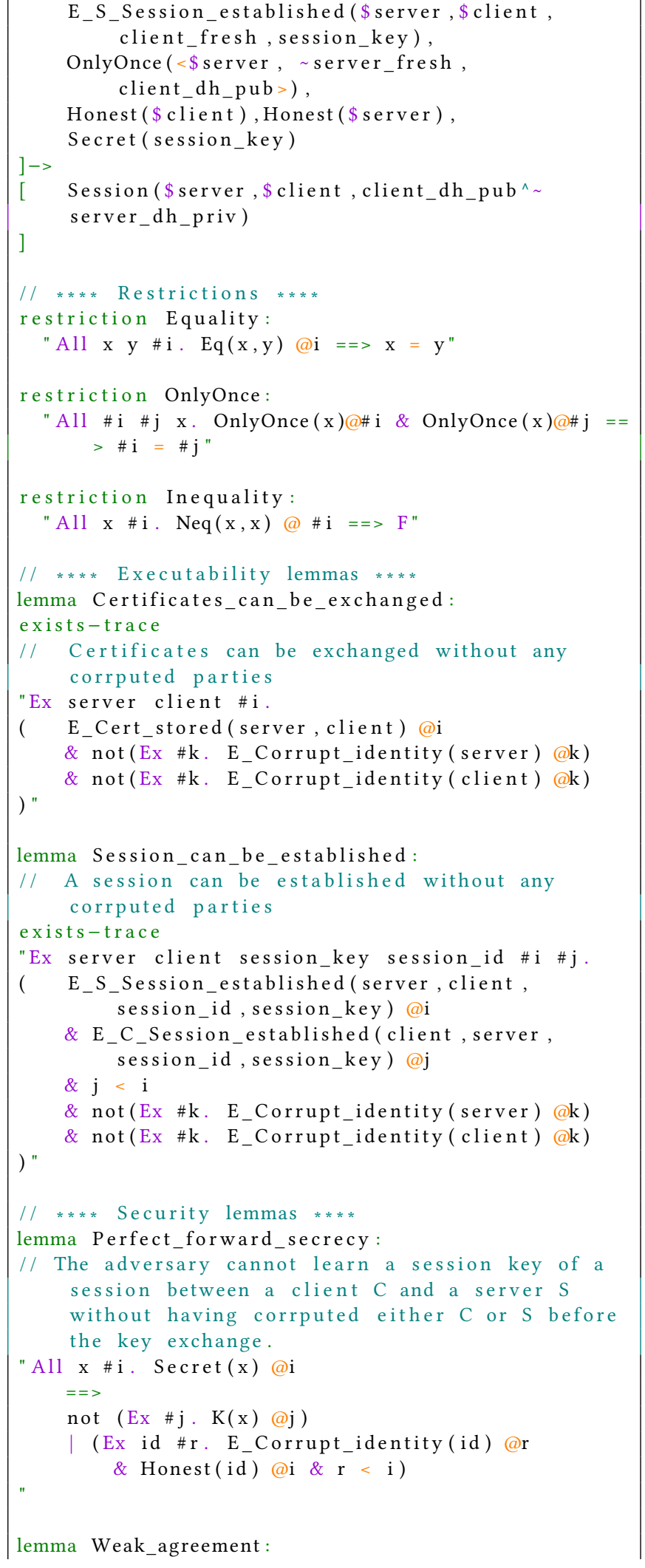

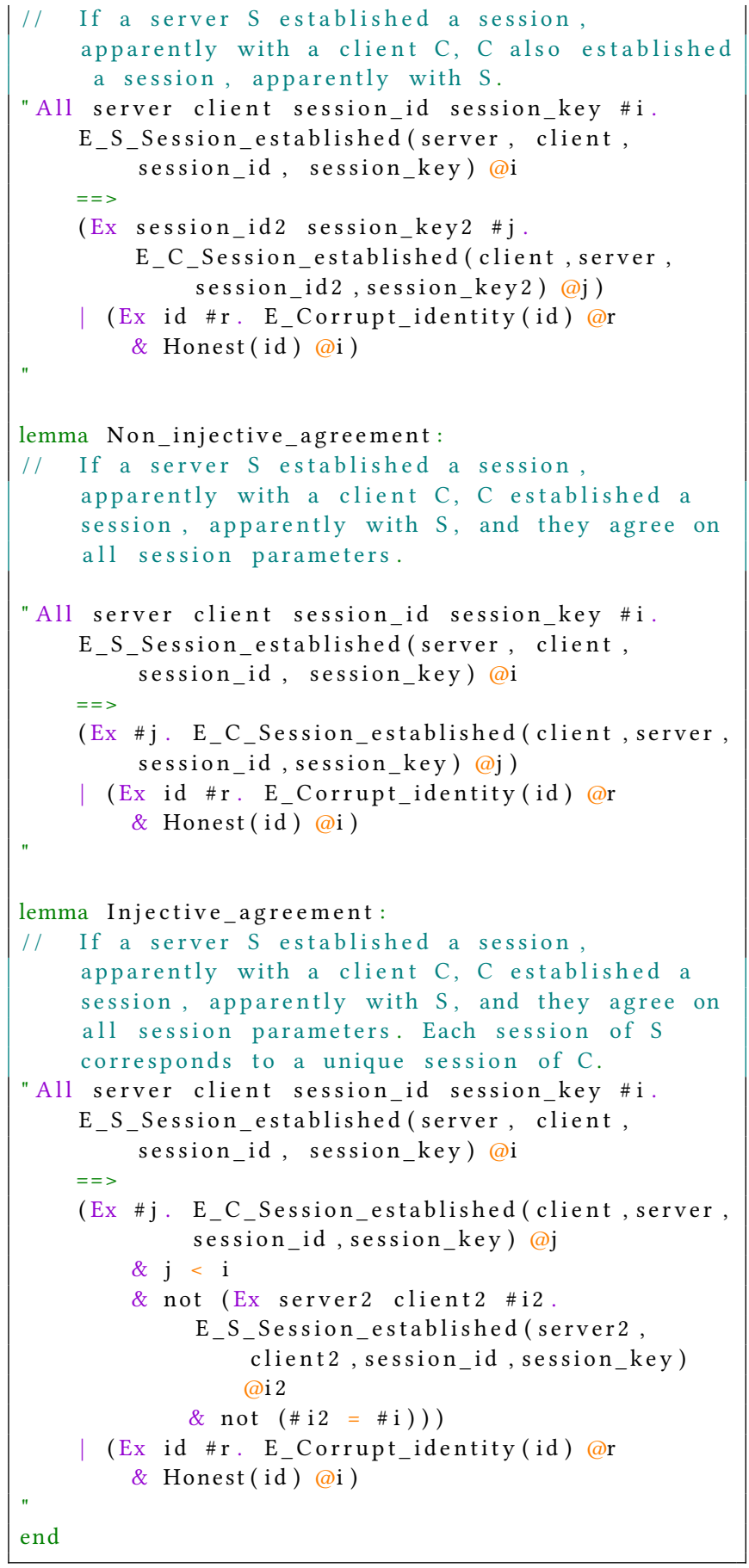

\section{A.3 Tamarin model of SESO-AS}

In our model of SESO-AS, freshness values have been abstracted by nonces. Moreover, the broadcast of service offer messages by the AS is represented by individual messages per client.

theory SESOas 


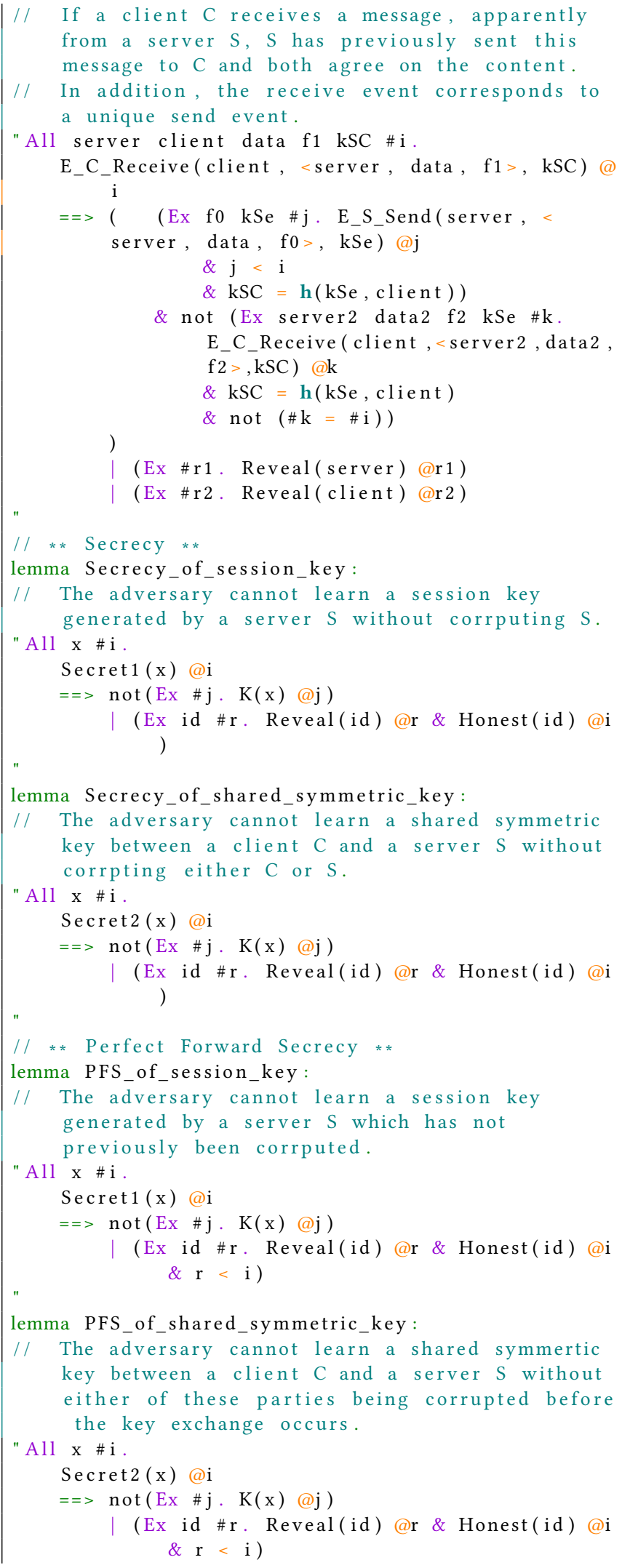

